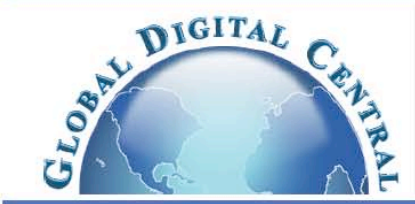

\title{
Frontiers in Heat and Mass Transfer
}

Available at www.ThermalFluidsCentral.org

\section{CHAOTIC NATURAL CONVECTION IN AN ANNULAR CAVITY WITH NON-ISOTHERMAL WALLS}

\author{
H.E. Dillon, A.F. Emery ${ }^{\dagger}$, A.M. Mescher, O. Sprenger, S.R. Edwards \\ University of Washington, Seattle, WA, 91805, US
}

\begin{abstract}
The stability of free convection in an annulus is governed by the boundary conditions on the inner and outer walls of the annulus and the upper and lower boundaries. This paper explores the effect of free convection on the inner surface of the annulus, where the boundary conditions for the outer wall and the upper and lower boundaries are controlled. The temperature is measured in the center of the air cavity and just below the surface of the inner annular boundary. Experimental results are shown for a radius ratio of 0.40 , aspect ratio of 20.7. These more recent experimental results are compared to prior work for a radius ratio of 0.60 , aspect ratio of 31.0. The results indicate that as the Rayleigh number is increased, the flow experiences a transition from steady flow to a very regular oscillatory flow, and then a supercritical Hopf bifurcation as the flow finally transitions to chaotic behavior. Proper orthogonal decomposition analysis is presented as a method for quantifying the complex dynamic behavior of the system.
\end{abstract}

Keywords: natural convection, dynamic system, experiment, annulus, chaos

\section{INTRODUCTION}

This paper describes the relationship between the flow stability of free convection motion in an annular geometry and the non-isothermal wall boundary conditions that drive it. The problem investigated here bears some similarity to the manufacturing process for drawing polymer fiber in which a polymer preform (cylindrical rod) is heated in a cylindrical furnace with a consequent non-uniform temperature at the moving polymer surface. In contrast to the drawing process for glass fiber where radiant heat transfer dominates and convective heat transfer is much less, convective heat transfer in polymer fiber drawing contributes roughly half of the total, and so it strongly influences the fiber diameter history. For this reason, an understanding of gas flow instabilities is critical, whether it is for the purpose of eliminating gas flow oscillations to produce constant diameter fiber, or enhancing oscillations to achieve controllable variations in fiber diameter.

The annular geometry for this study is shown in Fig. 1. The inner surface of the annulus is the stationary surface of a polymer preform. The surface temperature of the polymer is dependent on the radiant heat transfer from the furnace wall, the convective heat transfer from the natural convection cells in the annulus, as well as conduction heat transfer within the polymer preform. The upper and lower boundaries of the annulus are tightly fitting irises made of copper, which are each temperature controlled. Experiments have shown that the flow stability is primarily controlled by the relationship between the upper iris temperature and the furnace wall temperature. Further details of the experimental system are provided by Dillon et al. (2009).

The temperature differences between the vertical surfaces and the upper boundary drive a buoyant flow with the formation of multiple convection cells. The temperature oscillations of the convection cells were measured with thin wire thermocouples located in the air cavity. The effect of the convection cells was measured with thermocouples embedded just below the polymer surface. The temperature oscillations were then characterized by spectral analysis to determine the frequencies.

For natural convection studies in an annulus several parameters are used to classify the systems. The geometry of the air cavity is represented by the aspect ratio $(A)$ and the radius ratio $(\eta)$.

$$
\begin{gathered}
A=\frac{H}{R_{o}-R_{i}} \\
\eta=\frac{R_{i}}{R_{o}}
\end{gathered}
$$

where $R_{o}$ is the outer radius of the annulus, $R_{i}$ is the inner radius of the annulus, and $H$ is the height of the annulus. For the experimental system described here, the radius ratio is $\eta=0.40$ and the aspect ratio is $A=20.7$.

\section{BACKGROUND}

\subsection{Natural Convection}

The majority of natural convection studies in an annulus or two-dimensional rectangular cavity have been with simplified thermal boundary conditions, most typically with isothermal vertical walls. A review of twodimensional natural convection in the rectangular geometry is provided in Dillon et al. (2009).

\footnotetext{
${ }^{\dagger}$ Corresponding author.Email: emery@u.washington.edu
} 
Table 1 Summary of experimental natural convection studies in vertical annuli. Adapted from Dillon et al. (2009).

\begin{tabular}{|c|c|c|c|c|c|}
\hline Author & $\mathbf{R a}$ & Pr & $\eta$ & A & Description \\
\hline Choi and Korpela (1980) & $5 e 3-2 e 4$ & 0.71 & 0.68 & 38.6 & $\begin{array}{l}\text { Smoke photos with wavenumber of } 2.72 \text { reported. } \\
\text { Critical Grashof of } 9100 \text { reported. }\end{array}$ \\
\hline Stork and Muller (1975) & $2 e 3-4 e 3$ & 0.71 & 0.25 & $1.4-6.4$ & Smoke visualization of the flow. \\
\hline Keyhani et al. (1983) & $10 e 3-10 e 6$ & 0.71 & 0.23 & 27.6 & Also considered Helium. \\
\hline Weidman and Mehrdadtehranfar (1983) & $10 e 4-10 e 6$ & $15-150$ & 0.62 & 64 & Critical Ra based on photos $\left(R a_{c}=1-1.33 e 5\right)$. \\
\hline
\end{tabular}

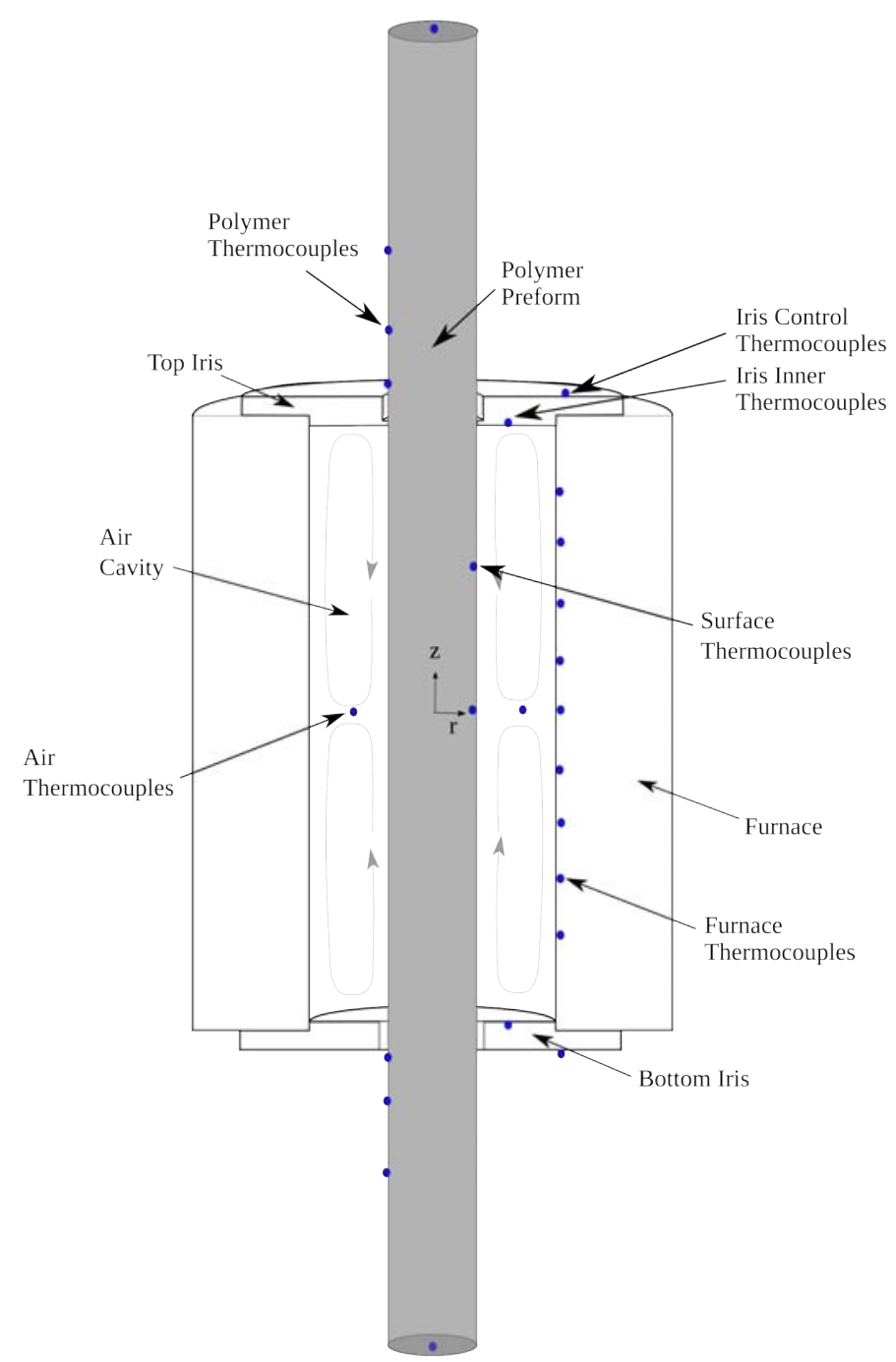

Figure 1 Diagram of the natural convection experiment. Thermocouple locations are shown as dots in the furnace wall and polymer preform.

The stability of flow in an annular cavity with isothermal vertical walls is governed by the Prandtl number (Pr), the Rayleigh number (Ra), and the geometry. At small Rayleigh numbers the flow is steady. As the Rayleigh number is increased the flow becomes unstable, first resulting in multicellular secondary flow patterns, and then as the Rayleigh number is further increased the flow becomes chaotic. The critical Rayleigh number $\left(R a_{c}\right)$ is used in buoyancy flows to characterize the transition between steady flow and the onset of oscillatory flow.

$$
\begin{gathered}
R a=\frac{\rho^{2} g c_{p} \beta(\Delta T) H^{3}}{k \mu} \\
\operatorname{Pr}=\frac{c_{p} \mu}{k}
\end{gathered}
$$

where $c_{p}$ is the specific heat at constant pressure, $g$ is the acceleration of gravity, $\beta$ is the isobaric coefficient of thermal expansion, $\mu$ is the dynamic viscosity, $k$ is the thermal conductivity, and $\rho$ is the density. By convention in the literature $\Delta T$ is defined as the difference between the temperatures of the two isothermal vertical walls. Most studies of annular regions have treated a heated inner wall and a cooled outer wall.

$$
\Delta T_{\text {literature }}=T_{\text {innerwall }}-T_{\text {outerwall }}
$$

In addition to numerical simulations, there have also been a number of experimental investigations of natural convection in annular cavities as summarized in Table 1, including this work. None of the prior experimental studies address the case of a non-isothermal outer wall.

\subsection{Proper Orthogonal Decomposition}

Reduced order models have become popular in recent years as a tool for understanding dynamic systems, particularly those involving complex fluid behavior. Proper orthogonal decomposition (POD) is a mathematical technique for reducing the dimensionality of a data set consisting of a large number of related variables while retaining as much information as possible about the data. In complex dynamic systems POD may be used to aid analysis of results and quantify the dynamic behavior of the system.

Proper Orthogonal Decomposition (POD) has many names and is also known as Karhunen-Louve Decomposition and principle component analysis (PCA). POD is broadly the Singular Value Decomposition (SVD) of a data matrix, which is typically normalized, Trefethen and Bau (1997). The POD may be computed using three different equivalent techniques as shown by Liang et al. (2002) but the SVD technique will be used in this work.

In the SVD calculation each principle component is a linear combination of the original variables and all principle components are orthogonal to each other so there is no redundant information. The first principal component is calculated as a single axis in space. When each observation is projected onto that axis the resulting values form a new variable. The second principal component is another axis in space perpendicular to the first. The principle components form an orthogonal basis for the space of the data and it is common for the sum of the first few principle components to exceed 90 percent of the total variance in the system. 
Table 2 Summary of POD modeling for dynamic fluid and heat transfer systems.

\begin{tabular}{|c|c|c|}
\hline Author & Analysis Type & Problem Overview \\
\hline Sirovich et al. (1991) & POD & Full channel flow. \\
\hline Bonnet et al. (1994) & POD LSE & Axisymmetric jet shear layer. \\
\hline Tarman (1996) & POD Galerkin & Rayleigh-Benard Convection. \\
\hline Liakopoulos et al. (1997) & POD Galerkin & Convection in a tall cavity. \\
\hline Webber et al. (1997) & POD & Minimal channel flow. \\
\hline Ly and Tran (2001) & POD & Rayleigh-Bernard Convection. \\
\hline Citriniti and George (2000) & POD & Axisymmetric mixing layer. \\
\hline Park and Jung (2001) & POD Galerkin & Inverse natural convection. \\
\hline Podvin and Quere (2001) & POD & Convection in a tall cavity. \\
\hline Sahan (2000) & POD Galerkin & $\begin{array}{l}\text { Natural convection } \\
\text { in a vertical channel }\end{array}$ \\
\hline Blinov et al. (2004) & POD MPS & Conduction \\
\hline Tarman (2003) & POD Galerkin & Rayleigh-Benard Convection. \\
\hline \multirow[t]{2}{*}{ Asokan and Zabaras (2005) } & POD & Rayleigh-Benard Convection. \\
\hline & Polynomial Chaos & \\
\hline
\end{tabular}

POD has been used for characterization of fluid systems by a number of investigators, particularly for turbulent or chaotic systems, as in Homes et al. (1996) and Park and Jung (2001). Table 2 provides a summary of some of the literature for this type of problem.

Several of the authors working on reduced order models included experimental results. Bonnet et al. (1994) combined the POD technique with Linear Stochastic Estimation (LSE) to compare experimentally obtained velocity measurements with their model. Citriniti and George (2000) used 138 hot-wire anemometers to gather the velocity field used for their POD analyses.

A few papers in the literature have discussed the possibility that the number of modes required to represent the system may correspond to the order of the dynamic system. Ties of this nature have been made by Ball et al. (2005) whose eigenvalue calculations (approximately 500) correspond well with the Lyapunov dimension calculations. Homes et al. (1996) suggests that for this type of analysis the number of modes retained should represent 90 percent of the energy in the system. However in previous work by the current authors, more than $99 \%$ of the energy had to be captured by the modes in order to give an accurate representation of the flow in an annular region.

Several POD models were developed for natural convection in a tall cavity. Liakopoulos et al. (1997) considered $A=40$ and Podvin and Quere (2001) considered $A=4$; however, the POD was based on a computational model of an air cavity rather than experimental results.

\section{WALL TEMPERATURE PROFILES}

A key characteristic of the experimental system is the outer wall temperature profile. Fig. 2 is an example of a furnace wall temperature profile that results in chaotic flow. In contrast to the conventional definition of $\Delta T$, the flow behavior observed experimentally has been better characterized by defining $\Delta T$ to be the difference between the peak wall temperature $\left(T_{\text {peak }}\right)$ and the upper iris temperature $\left(T_{\text {iris }}\right)$ where the upper iris temperature is the average temperature of the upper horizontal boundary of the cavity.

$$
\Delta T=T_{\text {peak }}-T_{\text {iris }}
$$

This definition correctly captures the expected trend, which is that a high Rayleigh number (high $\Delta T$ ) results in chaotic flow, whereas a low Rayleigh number (low $\Delta T$ ) results in steady flow. For some cases of steady flow, $T_{\text {iris }}$ can be higher than $T_{\text {peak }}$, in which case the $\Delta T$ defined in Equation 6 is negative and so also is the Rayleigh number.

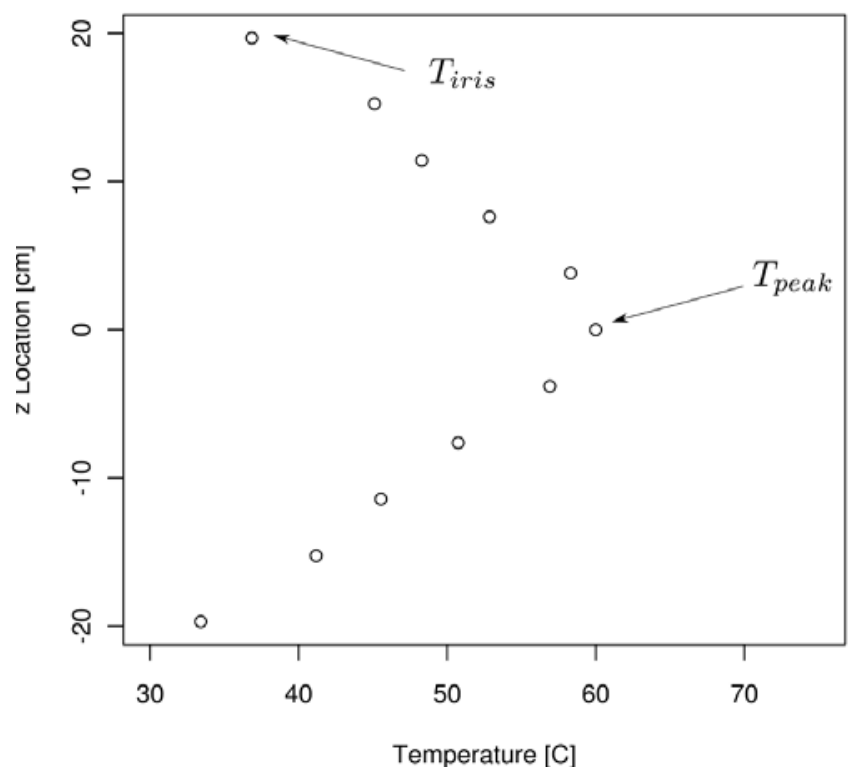

Figure 2 Wall temperature profile for chaotic behavior in the annular air cavity. $\eta=0.40, \Delta T=25^{\circ} \mathrm{C}$.

This definition of $\Delta T$ was chosen in contrast to the traditional definition $\left(\Delta T_{\text {literature }}\right)$ because for this work, the vertical walls have a nonuniform temperature distribution, the outer vertical surface of the annulus is warmer than the inner surface, and because the temperature difference with the upper iris $\left(T_{\text {iris }}\right)$ is what actually drives the buoyant behavior.

At high $\Delta T$ chaotic temperature oscillations occur in the air cavity and the wall profile is consistent with Fig. 2, where the top iris temperature is approximately $35^{\circ} \mathrm{C}$ and the peak wall temperature $\left(T_{\text {peak }}\right)$ is approximately $60^{\circ} \mathrm{C}$. As the upper iris temperature $\left(T_{\text {iris }}\right)$ is increased, oscillatory behavior occurs in the air cavity. As the $\Delta T$ is further decreased steady behavior occurs in the air cavity and the wall profile is characteristic of Fig. 3 where the peak wall temperature is now lower than the upper iris temperature, $60^{\circ} \mathrm{C}$ versus $71^{\circ} \mathrm{C}$ respectively. This results in a negative $\Delta T$; however, the behavior still shows the correct trend, where the lower $\Delta T$ 's indicate steady behavior and higher $\Delta T$ 's are increasingly oscillatory and then chaotic. 


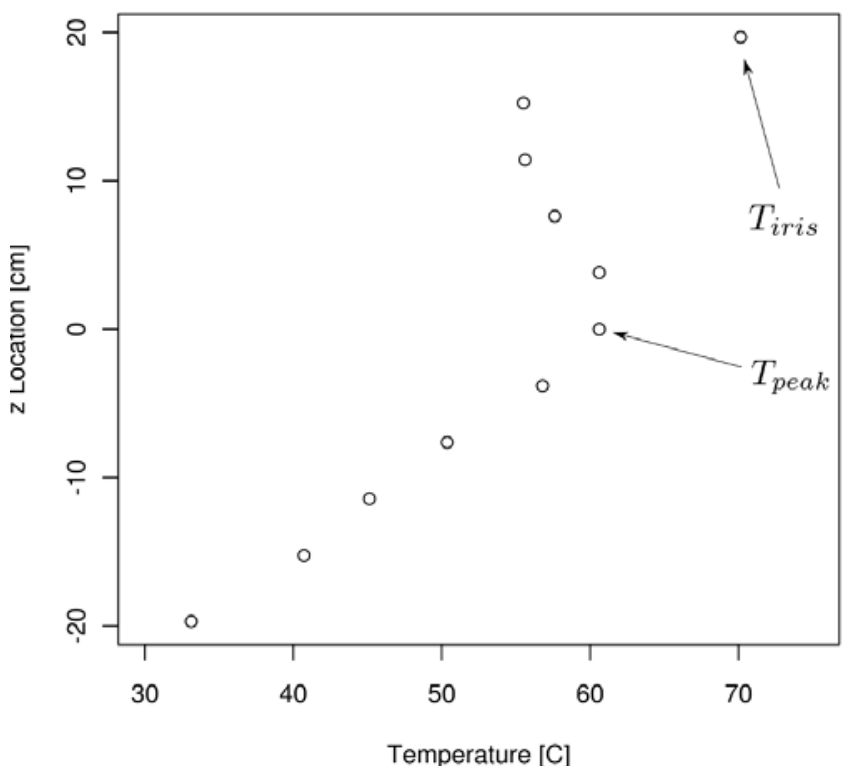

Figure 3 Wall temperature profile for steady behavior in the annular air cavity. $\eta=0.40, \Delta T=-11^{\circ} C$.

\section{EXPERIMENTAL SYSTEM}

Thin wire thermocouples $(0.125 \mathrm{~mm})$ are suspended in an annular cavity to record temperature oscillations in the air. Fig. 1 shows a schematic of the experimental system with the thermocouple locations. The four air thermocouples are spaced at $90^{\circ}$ azimuthal increments. Each of the air thermocouples in the furnace is designated by its azimuthal location, $0^{\circ}$, $90^{\circ}, 180^{\circ}$, and $270^{\circ}$.

Additional thermocouples are placed along the exposed, exterior surfaces of the polymer preform, the inner wall of the furnace, and at key locations including the inner surfaces of the copper irises. These thermocouples are used to determine the thermal boundary conditions, and to control the temperature on the vertical furnace wall, the upper horizontal boundary, and the lower horizontal boundary.

For the $\eta=0.40$ results presented in this paper, thin wire thermocouples were embedded just slightly below the acrylic (PMMA) preform surface at $z=0 \mathrm{~mm}$ and $50 \mathrm{~mm}$ (relative to the midheight of the annulus as shown in Fig. 1). This was achieved by: 1) machining a channel for the thermocouple wires, 2) tacking the wires into the channels, and 3) filling the channels with a 50/50 mixture of commercial adhesive and MMA. This mixture was chosen because it has similar thermal properties to the original acrylic, and the mixture has a longer drying time than pure MMA, which allowed the thermocouples to be placed properly.

Fig. 4 shows a cross-section of the acrylic preform with placement of the thermocouples at $180^{\circ}$ azimuthal offsets to provide duplication. The important dimensions for the channel milling are in the radial and azimuthal directions, with a depth of $0.254 \mathrm{~mm}$ (.01 inches) from the surface as shown in Fig. 4 (not to scale). Fig. 5 shows a completed channel with an embedded thermocouple.

The furnace has an inner height $(\mathrm{H})$ of $0.39 \mathrm{~m}$ (15.5 in), an inner diameter of $0.064 \mathrm{~m}$ (2.5 in), and is capped at both ends by irises. To control the vertical temperature profile along the furnace wall it is possible to apply power to any combination of 11 power taps located along the vertical height of the furnace. For these experiments power was applied across the mid-region of the furnace, $z=-5.5 \mathrm{~cm}$ to $z=5.5 \mathrm{~cm}$. This matches the conditions used to produce polymer fiber and results in a non-uniform temperature profile in the furnace wall. The top and bottom copper irises are each heated and independently controlled with thermocouples located on the outside surface of each iris.

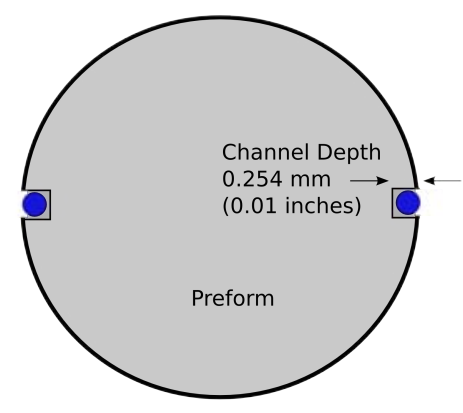

Figure 4 Cross-section of the polymer preform showing the embedded thermocouples. Not to scale.

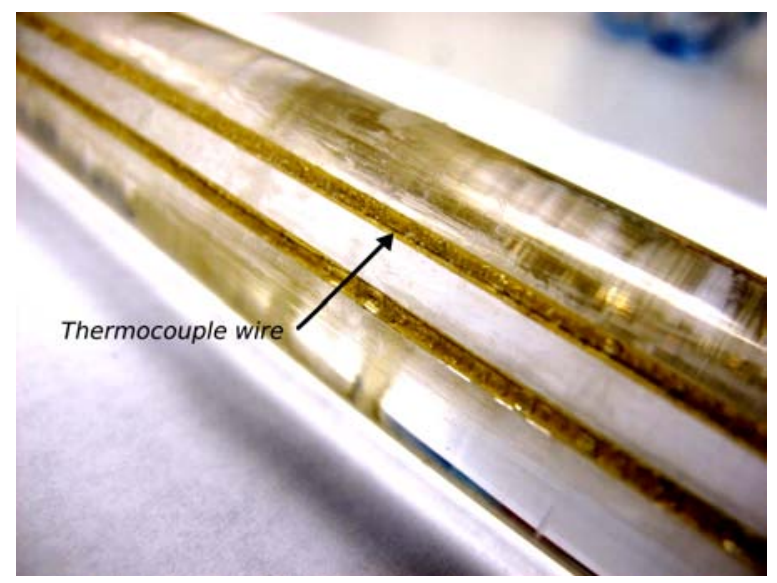

Figure 5 Close up of an acrylic preform with embedded thermocouple (wire).

An example of air temperature data for the radius ratio $\eta=0.60$ is shown in Fig. 6. The raw temperatures $\left(T_{\text {raw }}\right)$ are expressed as deviations from the temporal mean values $\left(T_{\text {mean }}\right)$ as the residual:

$$
\text { residual }=\frac{T_{\text {raw }}-T_{\text {mean }}}{T_{\text {mean }}}
$$

Fig. 7 illustrates the corresponding spectral density function (sdf) calculated using a Welch's Overlapped Segment Averaging (WOSA) method with 20 windows. Additional details about the spectral analysis and experimental procedure are included in the prior work, Dillon et al. (2009). For these experimental conditions the flow clearly shows a periodic behavior with near harmonic higher order frequencies.

\section{RESULTS FOR RADIUS RATIO $\eta=0.40$}

The observed flow for $\eta=0.40$ exhibits all flow regimes. This is in contrast to prior results for $\eta=0.60$ which did not exhibit chaotic behavior for the same imposed thermal boundary conditions. For $\eta=0.40$, the steady region exists for $\Delta T<-11^{\circ} C$ and the oscillatory region exists for $-11^{\circ} C<\Delta T<-5^{\circ} C$. A quasi-periodic region exists for $-5^{\circ} C<\Delta T<2^{\circ} C$. The region $\Delta T>2^{\circ} C$ is classified as chaotic.

At an iris temperature of $32^{\circ} \mathrm{C}\left(\Delta T=28^{\circ} \mathrm{C}\right)$ the system demonstrates characteristics of a truly chaotic flow. In this case chaotic behavior is defined by Stogatz (2000), where aperiodic indicates trajectories that do not settle to fixed points or periodic orbits. Chaotic flow is indicated visually by aperiodic temperatures as shown in Fig. 8 for $\eta=0.40$ and the $\Delta T=25^{\circ} C$ furnace condition. The corresponding spectral analysis in Fig. 9 indicates that no single frequency has a dominant intensity. 


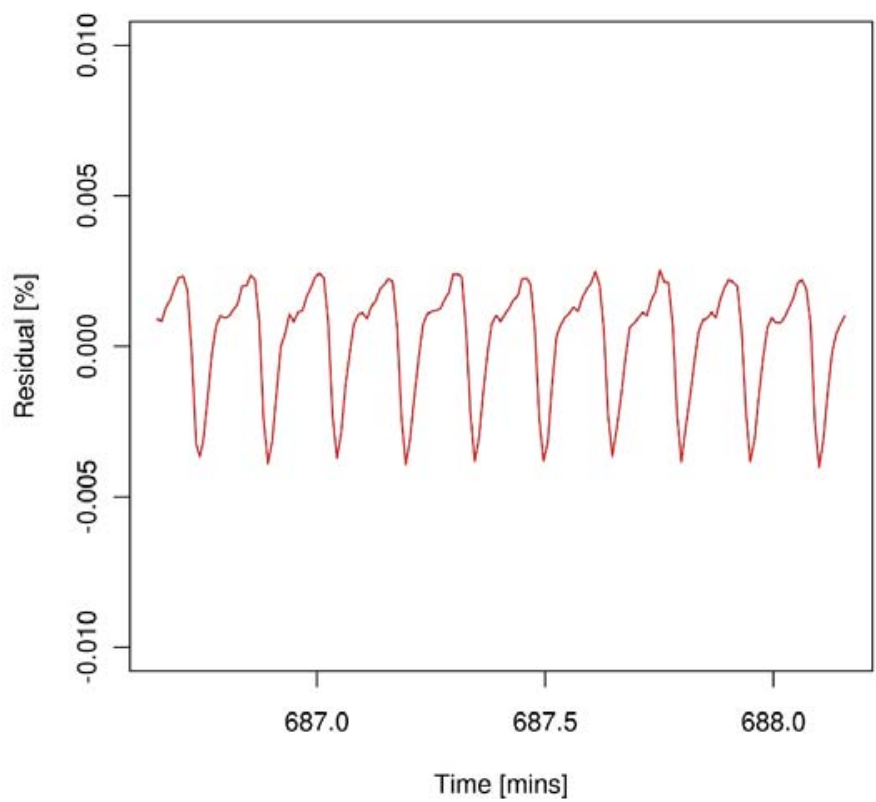

Figure 6 Air temperature residuals for the oscillatory regime of $\eta=$ 0.60. Results shown are for the thermocouple located at $270^{\circ} \mathrm{C}$ and the $\Delta T=25^{\circ} \mathrm{C}$ furnace condition. Adapted from Dillon et al. (2009).

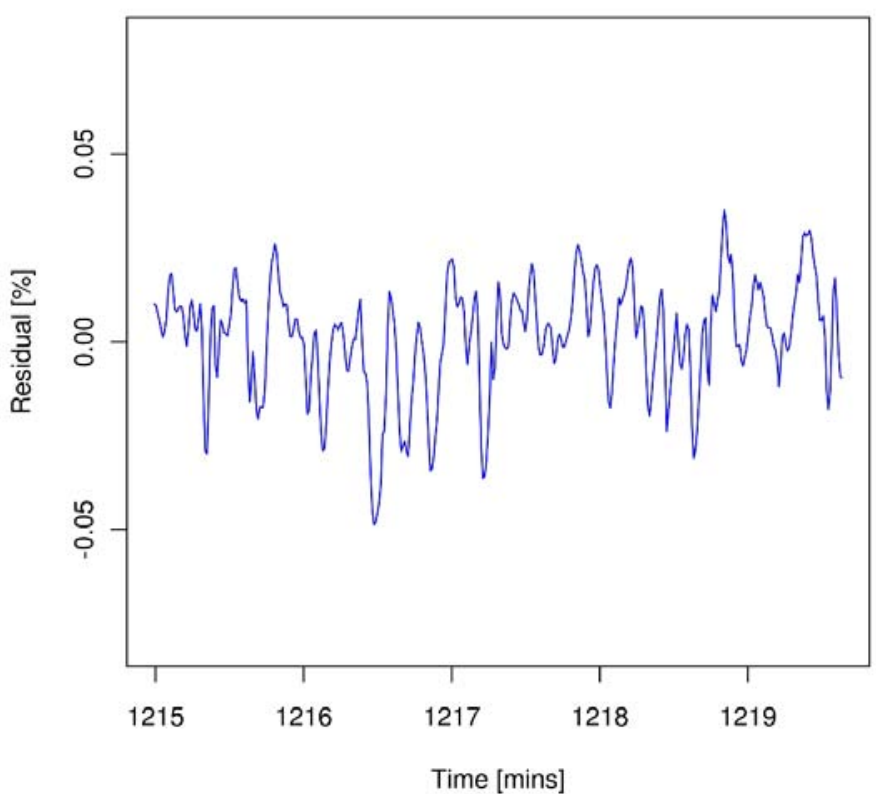

Figure 8 Air temperature residuals for the chaotic regime of $\eta=0.40$. Results shown are for the thermocouple located at $270^{\circ}$ and the $\Delta T=25^{\circ} \mathrm{C}$ furnace condition.

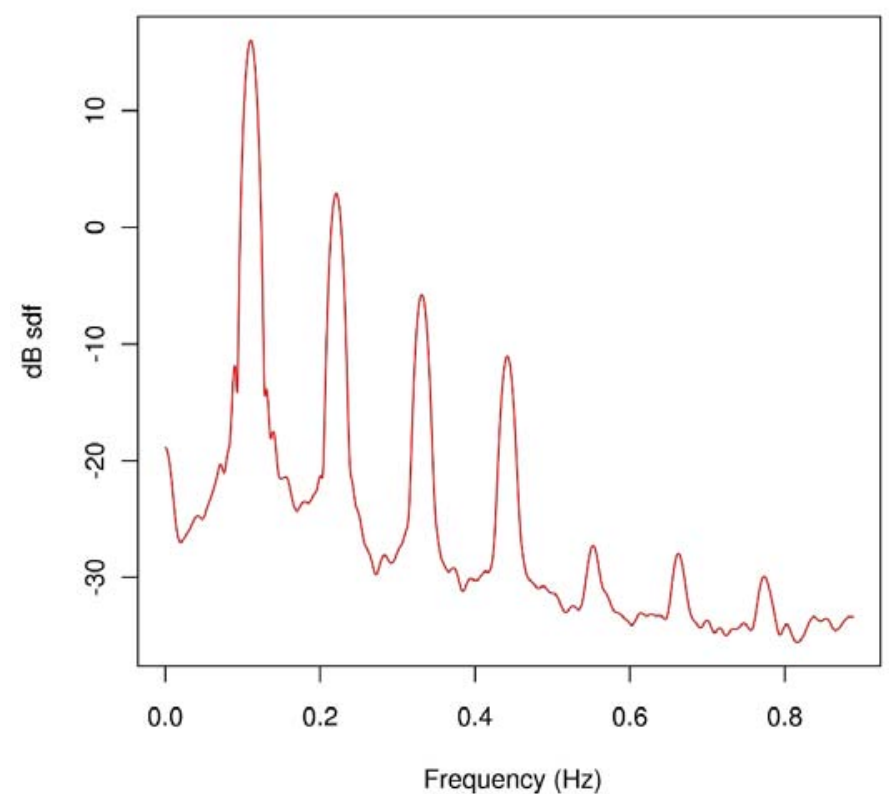

Figure 7 Air temperature frequencies for the oscillatory regime of $\eta=$ 0.60. The frequencies indicate that the system has strong oscillations near 0.1 and $0.2 \mathrm{~Hz}$. Results shown are for the thermocouple located at $270^{\circ} \mathrm{C}$ and the $\Delta T=25^{\circ} \mathrm{C}$ furnace condition. Adapted from Dillon et al. (2009).

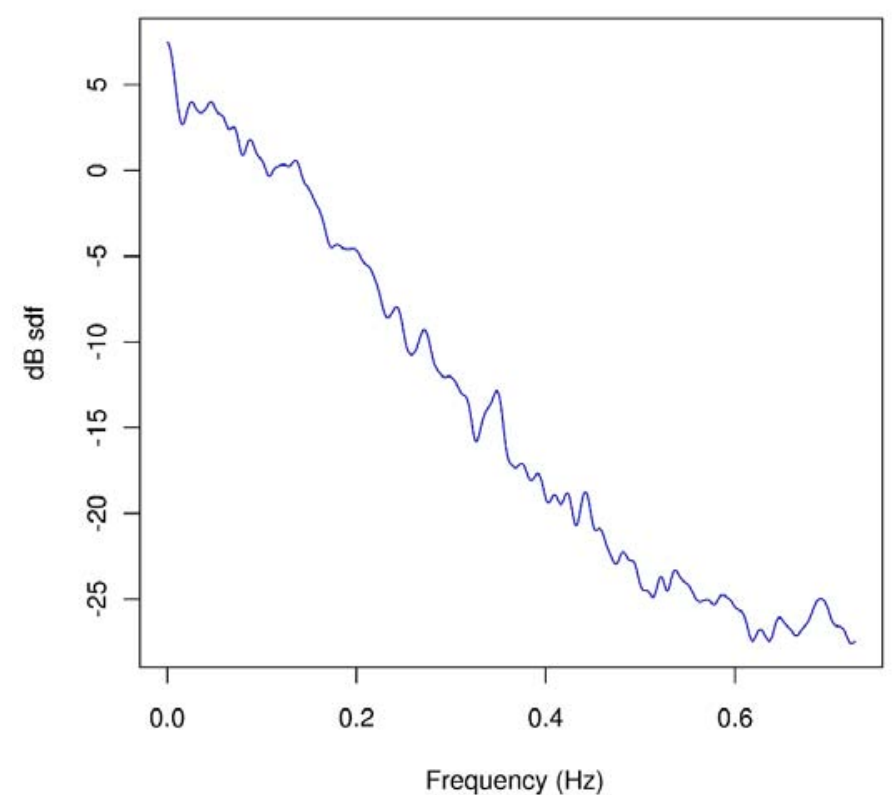

Figure 9 Air temperature frequencies for the chaotic regime of $\eta=0.40$. The frequencies indicate that the system is chaotic because no significant frequencies dominate. Results shown are for the thermocouple located at $270^{\circ}$ and the $\Delta T=25^{\circ} \mathrm{C}$ furnace condition. 
The quasi-periodic region for $\eta=0.40$ is shown in Fig. 10. A single peak frequency, shown in Fig. 11, occurs at $0.05 \mathrm{~Hz}$. Quasi-periodic behavior also occurs for the previously studied $\eta=0.60$, but in the case of $\eta=0.40$, the magnitude of the frequency has a much lower spectral power $(\mathrm{dB})$ by comparison.

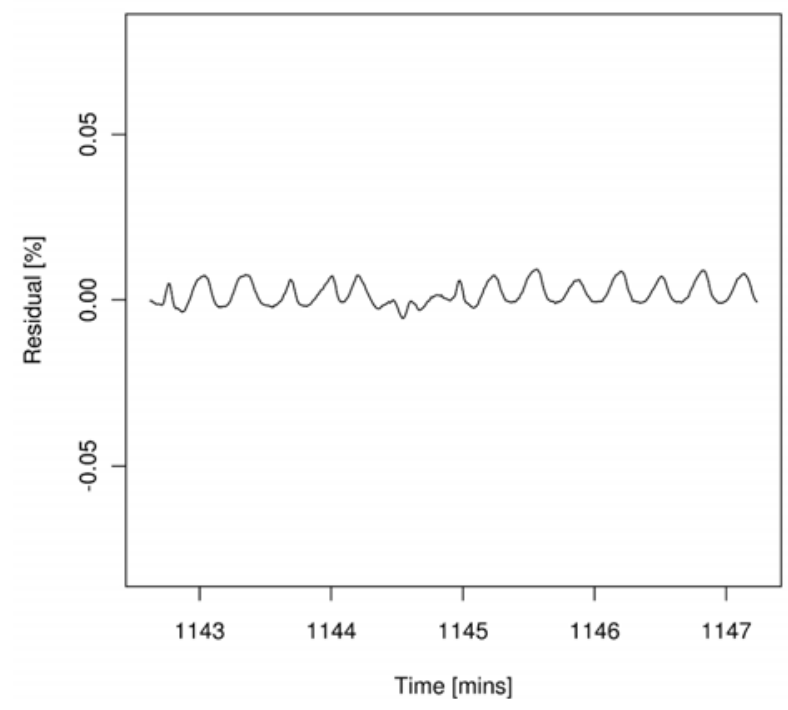

Figure 10 Air temperature residuals for the quasi-periodic regime of $\eta=$ 0.40 . Results shown are for the thermocouple located at $0^{\circ}$ and the $\Delta T=-1^{\circ} C$ furnace condition.

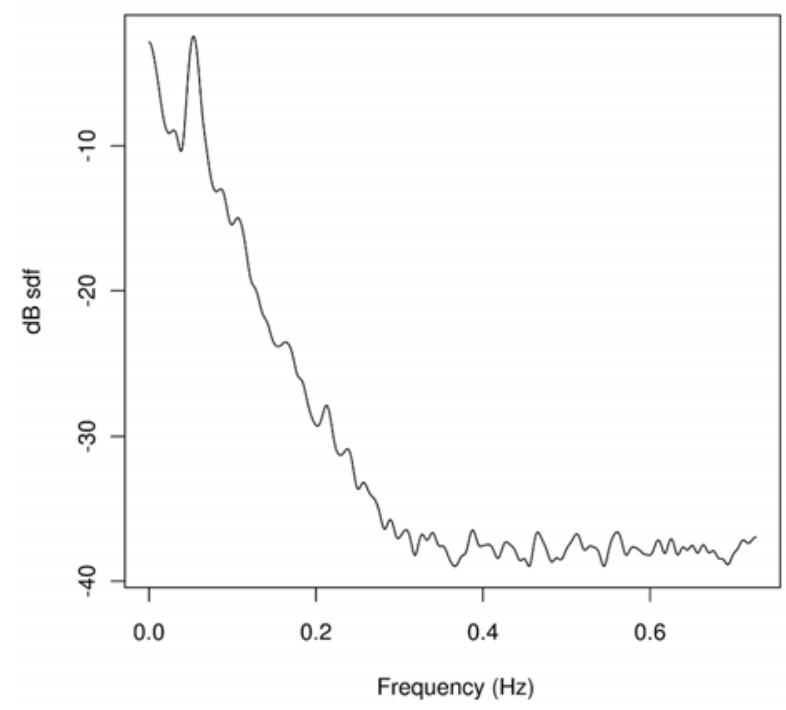

Figure 11 Air temperature frequencies for the quasi-periodic regime of $\eta=0.40$. A primary frequency of $0.05 \mathrm{~Hz}$ is observed. Results shown are for the thermocouple located at $0^{\circ}$ and the $\Delta T=-1^{\circ} C$ furnace condition.

The oscillatory region for $\eta=0.40$ also occurs, and an example is shown in Fig. 12 for the $\Delta T=-7^{\circ} C$ furnace condition. This oscillatory flow has primary frequencies at $0.06 \mathrm{~Hz}, 0.11 \mathrm{~Hz}$ (period halving), and $0.17 \mathrm{~Hz}$, as illustrated in Fig. 13. The amplitude of the oscillations is very small for these experimental conditions, and the frequencies have low spectral power compared to results from the previous studies with $\eta=0.60$.
As expected for the steady region, there is no significant variation in temperature and no frequencies observed in the frequency spectrum. An example is shown in Fig. 14 and Fig. 15 for the $\Delta T=-11^{\circ} C$ furnace condition.

\section{POLYMER SURFACE TEMPERATURE VARIATION}

For the application of drawing polymer fiber, an understanding of the relationship between air temperature and polymer temperature variations is important. In these experiments with an annular cavity, the temperature of the polymer (acrylic) preform is influenced by conduction within the stationary solid preform, radiation from the furnace wall, and gas phase convection in the annular cavity. Results from the thermocouples just below the surface of the preform indicate that the behavior of the air directly affects the polymer temperature, but as expected a lower amplitude of temperature variations occur. The temperatures just below the preform surface are correlated in time with the measured air temperatures.

In the quasi-chaotic regime the preform thermocouples show a quasichaotic variation with amplitudes an order of magnitude lower than those in the air. Fig. 16 shows the frequency spectrum of the preform temperature for the quasi-periodic regime, corresponding to the frequency spectrum of the air temperature shown in Fig. 11. Fig. 17 shows the frequency spectrum of the preform temperature for the oscillatory regime, corresponding to the frequency spectrum of the air temperature shown in Fig. 13. It is clear from comparison of the air and preform behavior that the surface of the preform is following the air effects, but with much lower spectral power.

\section{AIR TEMPERATURE VARIATIONS IN THE VERTICAL DIRECTION}

A subset of experiments was conducted to understand the complexity of the natural convection cells forming in the annular cavity. No visualization is possible due to the furnace construction but one of the thin wire thermocouples was traversed vertically $(z>0)$ to understand the changes in amplitude and mean temperature of the oscillations in the upper air cavity.

Fig.18 shows the air temperature variations as a function of vertical $z$ location for the chaotic regime of $\eta=0.40$. The dot indicates the mean temperature for each $z$ location and the bar indicates the standard deviation, representing the approximate amplitude of the oscillations. Large amplitude oscillations exist through most of the air cavity, and the mean air temperatures follow the general trend imposed by the non-isothermal furnace wall.

Fig. 19 shows the measured air temperature variations as a function of vertical $z$ location for the oscillatory regime of $\eta=0.40$, indicating the possible existence of multiple cells in the annular cavity. Multiple cells have been observed in previous work, including Reeve (2003), and Choi and Korpela (1980). Particle Imaging Velocimetry (PIV) also revealed the existence of multiple cells in a non-annular geometry that is more characteristic of the polymer fiber drawing environment, Reeve (2003).

\section{DYNAMIC SYSTEM BEHAVIOR OF $\eta=0.40$ AND COMPARISION TO $\eta=0.60$}

Here a comparison is made between results from the wider annular cavity of $\eta=0.40$ to a narrower cavity of $\eta=0.60$, previously investigated and reported in Dillon et al. (2009). It should be noted that in the previous study of $\eta=0.60$, a polycarbonate preform was used instead of acrylic as in the $\eta=0.40$ study. The advantage of a polycarbonate preform is that much higher furnace wall temperatures can be imposed (and therefore a greater range of $\Delta T$ 's), without risk of deformation of the preform; however, the advantage of an acrylic preform is that thermocouples can be conveniently embedded below the surface, using back-fill material (methyl methacrylate) with similar thermal characteristics to acrylic (polymethyl methacrylate). For this reason, polycarbonate temperatures 


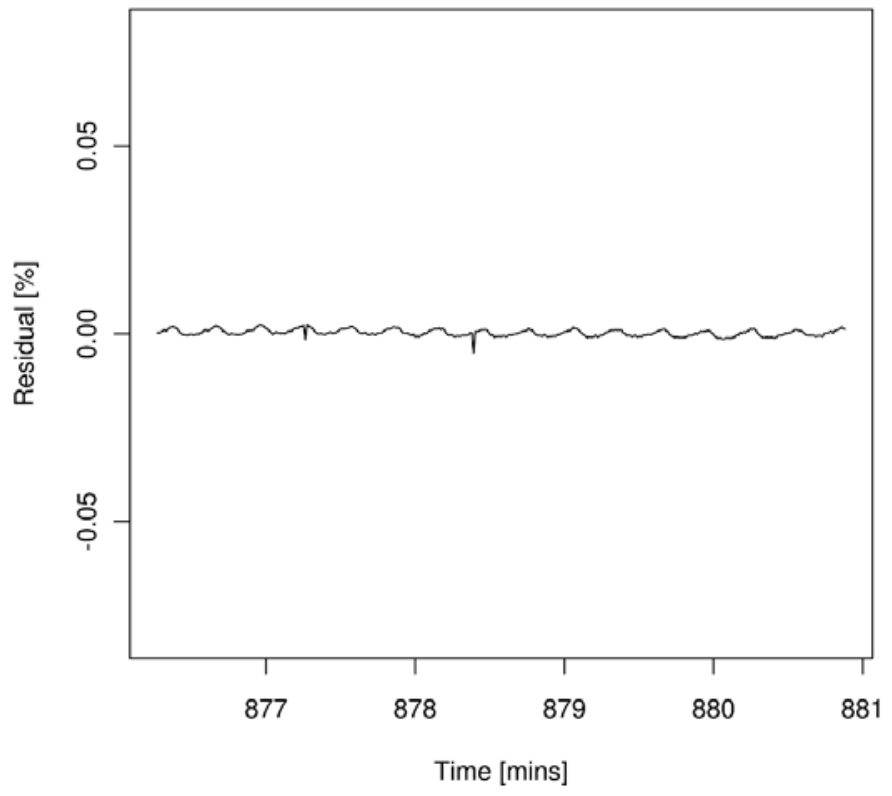

Figure 12 Air temperature residuals for the oscillatory regime of $\eta=$ 0.40 . Results shown are for the thermocouple located at $0^{\circ}$ and the $\Delta T=-7^{\circ} C$ furnace condition.

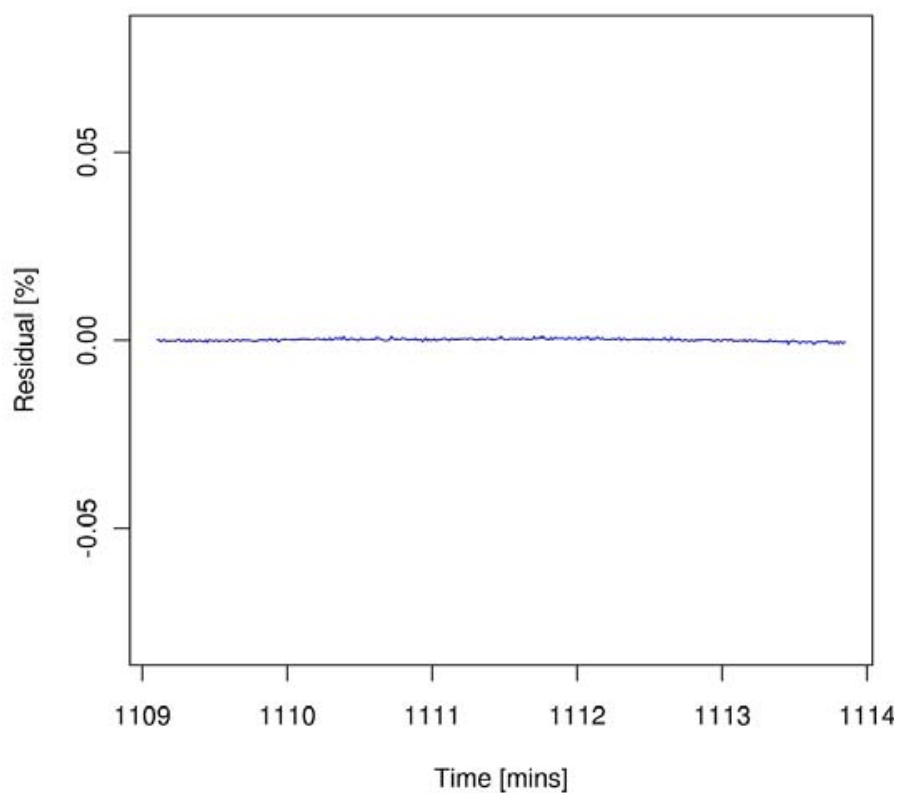

Figure 14 Air temperature residuals for the steady regime of $\eta=0.40$. Results shown are for the thermocouple located at $270^{\circ}$ and the $\Delta T=-11^{\circ} \mathrm{C}$ furnace condition.

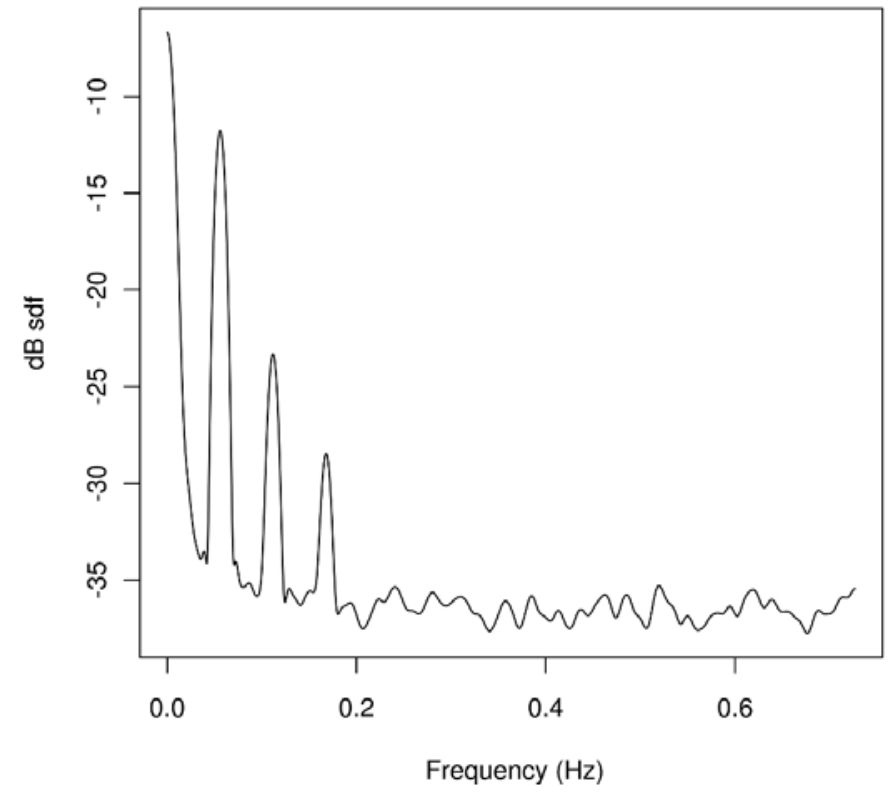

Figure 13 Air temperature frequencies for the oscillatory regime of $\eta=$ 0.40 . Primary frequency occur at $0.06 \mathrm{~Hz}, 0.11 \mathrm{~Hz}$, and 0.17 $\mathrm{Hz}$. Results shown are for the thermocouple located at $0^{\circ}$ and the $\Delta T=-7^{\circ} C$ furnace condition.

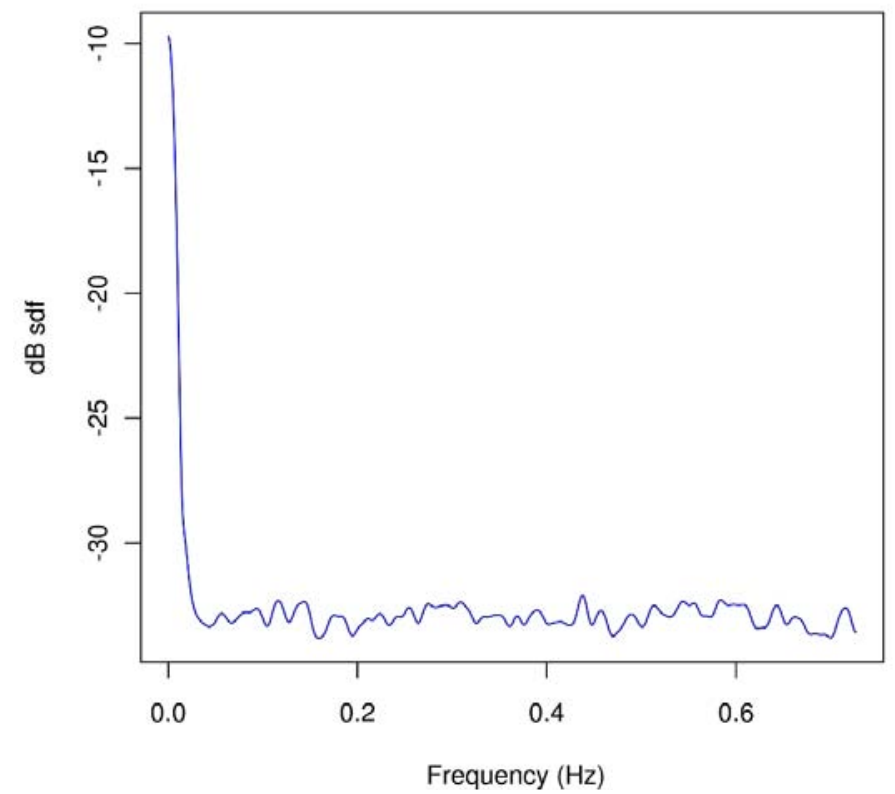

Figure 15 Air temperature frequencies for the steady regime of $\eta=$ 0.40. Results shown are for the thermocouple located at $270^{\circ}$ and the $\Delta T=-11^{\circ} C$ furnace condition. 


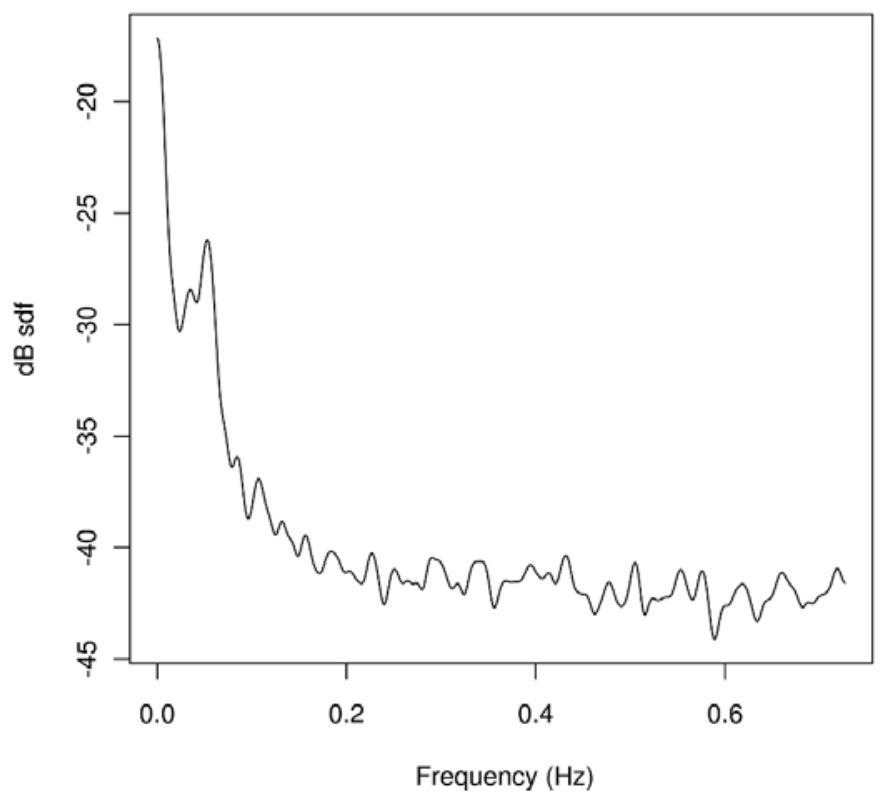

Figure 16 Preform temperature frequencies for the quasi-periodic regime of $\eta=0.40$. Results shown are for the thermocouple located in the preform at $z=0$, and the $\Delta T=-1^{\circ} C$ furnace condition. The frequency spectrum indicates that the preform surface has a similar response near $0.05 \mathrm{~Hz}$ to the air temperature frequency shown in Fig. 11.

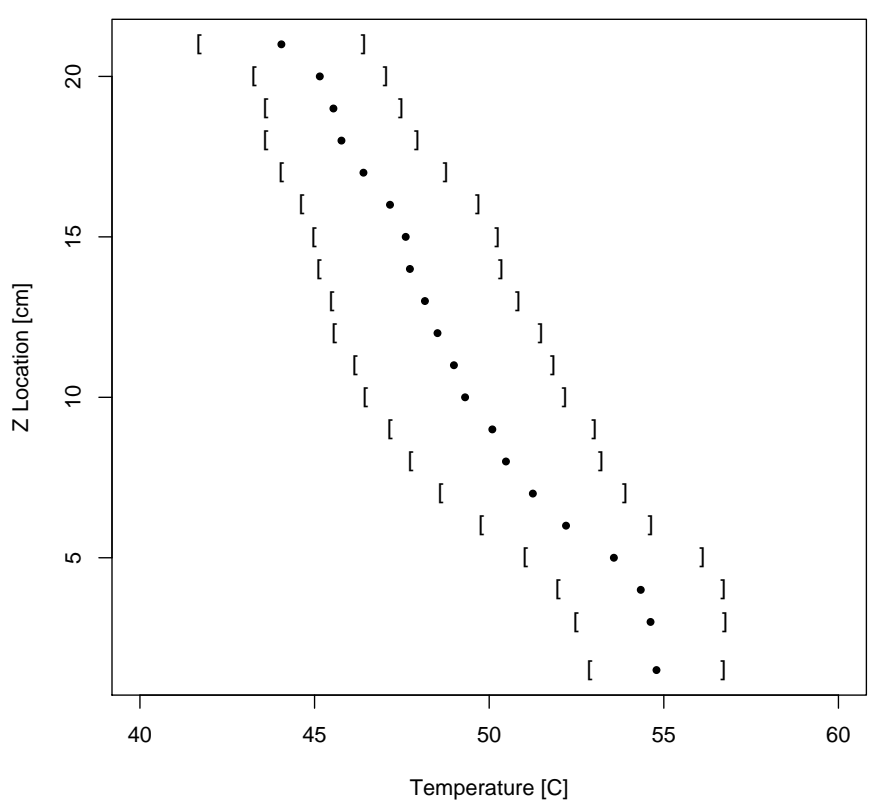

Figure 18 Air temperature variations as a function of vertical $z$ location for the chaotic regime of $\eta=0.40, \Delta T=25^{\circ} C$ furnace condition. The dot indicates the mean temperature for each $\mathrm{z}$ location, and the bar indicates one standard deviation, representing the approximate amplitude of the oscillations.

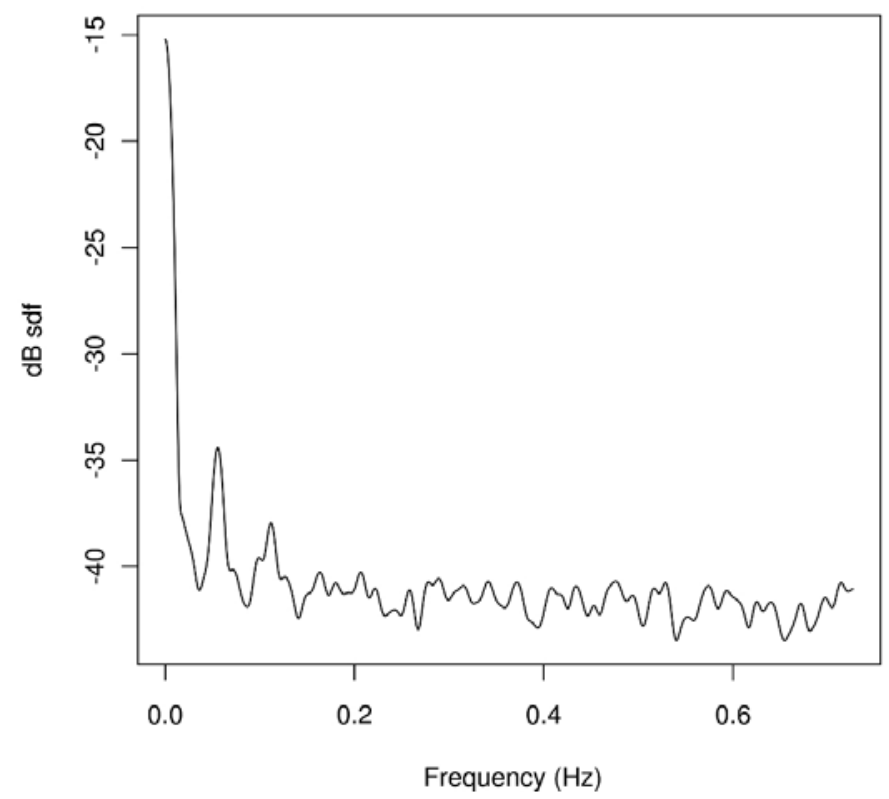

Figure 17 Preform temperature frequencies for the oscillatory regime of $\eta=0.40$. Results shown are for the thermocouple located in the preform at $z=0$, and the $\Delta T=-7^{\circ} C$ furnace condition. The frequency spectrum indicates that the preform surface has a similar response near $0.06 \mathrm{~Hz}$ and $0.11 \mathrm{~Hz}$ to the air temperature frequencies shown in Fig. 13.

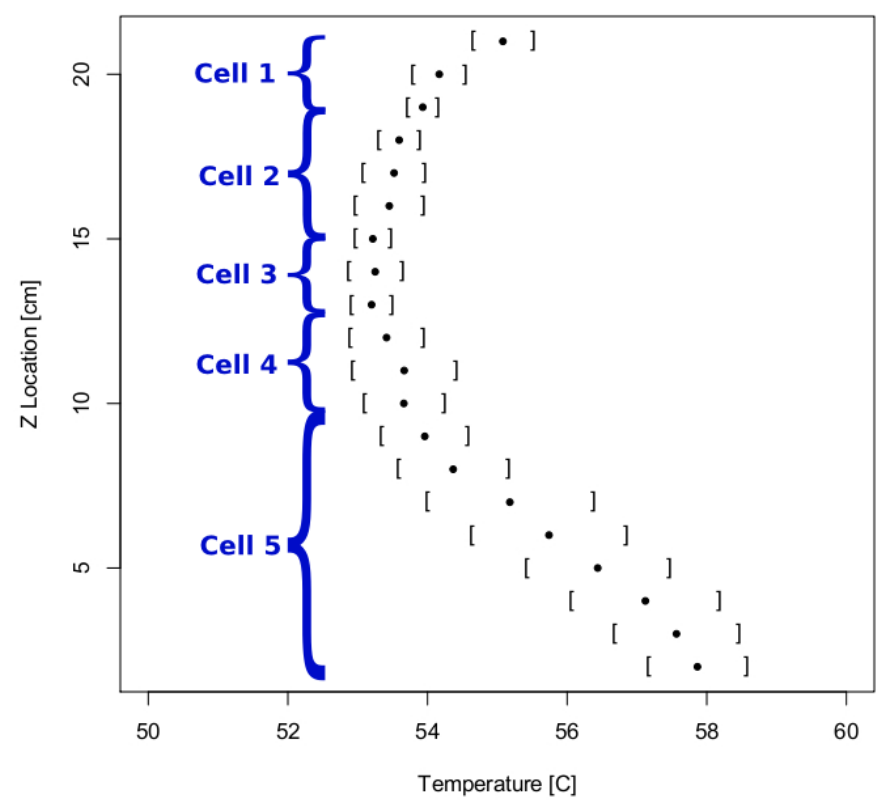

Figure 19 Air temperature variations as a function of vertical $\mathrm{z}$ location for the oscillatory regime of $\eta=0.40, \Delta T=-5^{\circ} C$ furnace condition. The dot indicates the mean temperature for each $\mathrm{z}$ location, and the bar indicates one standard deviation, representing the approximate amplitude of the oscillations. 
were not measured in the previous $\eta=0.60$ study. Acrylic preform temperatures were measured in the $\eta=0.40$ study, as given in Section 6 of this paper, but these temperature variations occurred at relatively low amplitude due in part to the much lower $\Delta T$ 's imposed in the thermal boundary conditions for $\eta=0.40$ compared to $\eta=0.60$. Since only air temperatures were measured for both $\eta=0.40$ and $\eta=0.60$, these measurements provide the basis of comparison for convection in the two annular geometries.

Table 3 summarizes, for $\eta=0.40$ and $\eta=0.60$, the air temperature frequencies and amplitudes observed for each of the flow regimes (chaotic, quasi-periodic, and oscillatory). The first row entries of Table 3 are the range of $\Delta T$ 's imposed to produce each of the flow regimes, including the steady flow regime. In the $\eta=0.60$ configuration, the system did not reach a fully chaotic regime. To reach a fully chaotic regime, active cooling of the upper iris would have been required. For $\eta=0.40$ and an imposed $\Delta T$ between $28^{\circ} C<\Delta T<2^{\circ} C$, the system is fully chaotic (as shown in Fig. 8 for $\Delta T=25^{\circ} C$ ).

Table 3 Summary of imposed $\Delta T$ ranges, measured air temperature amplitudes and frequencies, for the wider $(\eta=0.40)$ and narrower annular air cavities $(\eta=0.60)$. The reported amplitudes are an average of the four thermocouples in the air cavity. The experimental results for the narrower air cavity are documented in Dillon et al. (2009).

\begin{tabular}{|l|l|l|l|l|}
\hline Description & Chaotic & $\begin{array}{l}\text { Quasi- } \\
\text { periodic }\end{array}$ & Oscillatory & Steady \\
\hline \hline$\Delta T\left[{ }^{\circ} C\right]$ & 28 to 2 & $\begin{array}{l}2 \text { to }-5 \\
45 \text { to } 29\end{array}$ & -5 to -11 & $\Delta T<-11$ \\
$\eta=0.40$ & - & & & $\Delta T<11$ \\
$\eta=0.60$ & 2.05 & 0.33 & 0.16 & \\
\hline Amplitude $\left[{ }^{\circ} C\right]$ & & 4.12 & 0.74 & \\
$\eta=0.40$ & - & & & \\
$\eta=0.60$ & & & & \\
\hline Primary & & 0.05 & $0.06,0.11$ & \\
Frequencies $[\mathrm{Hz}]$ & & 0.05 & $0.11,0.22$ & \\
$\eta=0.40$ & & & & \\
$\eta=0.60$ & - & \multicolumn{2}{|l}{} \\
\hline
\end{tabular}

In comparison to the quasi-periodic regime of $\eta=0.40$ (Fig. 10 and Fig. 11), a much larger $\Delta T$ must be imposed to obtain the quasi-periodic regime of $\eta=0.60$, as shown in Table 3. The resulting amplitude of air temperature variations for $\eta=0.60$ is much greater than that for $\eta=0.40$ (4.12 to 0.33 ). The air temperature frequencies however for the two geometries are practically the same at $0.05 \mathrm{~Hz}$, in their respective quasi-periodic regimes.

The oscillatory regime of $\eta=0.40$ (Fig. 12 and Fig. 13) may be compared to the oscillatory regime of $\eta=0.60$ (Fig. 6 and Fig. 7). Again as shown in Table 3, a much larger $\Delta T$ must be imposed to obtain the oscillatory regime of $\eta=0.60$ than that required to generate regular oscillations for $\eta=0.40$. Also, the oscillatory air temperature amplitude for $\eta=0.60$ is greater than that for $\eta=0.40$ ( 0.74 to 0.16$)$. The air temperature frequencies for the oscillatory regimes of the two geometries are distinctly different as listed in Table 3 , and as illustrated by a comparison of Fig. 13 (for $\eta=0.40$ ) and Fig. 7 (for $\eta=0.60$ ).

For a constant peak wall temperature of $T_{\text {peak }}=80^{\circ} \mathrm{C}$ in the $\eta=$ 0.60 study, Fig. 20 shows recorded air temperature frequencies as a function of the top iris temperature. Starting in the oscillatory regime at the right side of the plot with $\Delta T=\left(T_{\text {peak }}-T_{\text {iris }}\right)=80-65^{\circ} \mathrm{C}=15^{\circ} \mathrm{C}$, three frequencies are clearly visible. As the top iris temperature is decreased to $55^{\circ} \mathrm{C}$, with $\Delta T=25^{\circ} \mathrm{C}$, these three characteristic frequencies are still clearly visible (shown also by the three highest peaks in Fig. 7). Near $T_{\text {iris }}=55^{\circ} \mathrm{C}$, the frequencies bifurcate and the system then approaches a chaotic condition as $T_{\text {iris }}=51^{\circ} \mathrm{C}$ and $\Delta T=29^{\circ} \mathrm{C}$.

In the $\eta=0.40$ study, with a constant peak wall temperature of $T_{\text {peak }}=60^{\circ} \mathrm{C}$, the results are less clear because the system becomes chaotic very quickly near $T_{\text {iris }}=58^{\circ} \mathrm{C}\left(\Delta T=2^{\circ} \mathrm{C}\right)$. Nevertheless, the same general behavior is illustrated in Fig. 21 as in Fig. 20. For both geometries, with $\eta=0.40$ and $\eta=0.60$, the systems undergo a frequency doubling route to chaos, consistent with other convection systems Homes et al. (1996). The transition from the steady regime to the oscillatory regime occurs as a supercritical Hopf bifurcation, also consistent with the results of other authors who have studied natural convection for $\Delta T$ imposed across two isothermal vertical walls.

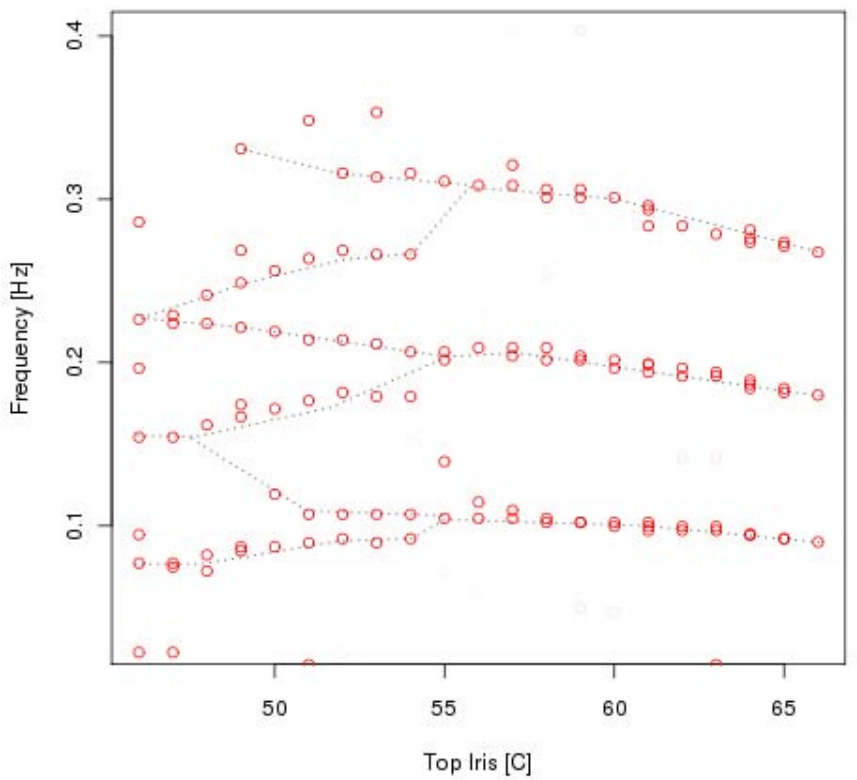

Figure 20 Frequencies measured for $\eta=0.60$ over a range of $\Delta T$ 's (Rayleigh number). Dotted lines have been added to emphasize frequency bifurcations near $T_{\text {iris }}=55^{\circ} \mathrm{C}\left(\Delta T=25^{\circ} \mathrm{C}\right)$.

\section{PROPER ORTHOGONAL DECOMPOSITION}

Computational fluid dynamic (CFD) simulation has been carried out for this experimental system and is reported in Dillon (2011). Non-isothermal boundary conditions, low buoyancy driving force, and long computation times make it computationally expensive to apply CFD simulation for practical industrial fiber drawing. For this reason a reduced order model of the experimental data has been developed to quantify the dynamic system behavior.

Assume a matrix $X$ is an $m \times n$ matrix composed of multiple observations from an experiment. In this case, each column represents measured temperatures at each time, and each row represents data collected for an imposed set of thermal boundary conditions with specified $\Delta T$.

$$
X=\left[\begin{array}{ccc}
T_{a}\left(t_{1}\right) & T_{a}\left(t_{2}\right) & \ldots \\
T_{b}\left(t_{1}\right) & T_{b}\left(t_{2}\right) & \ldots \\
\vdots & & \vdots \\
T_{m}\left(t_{1}\right) & T_{m}\left(t_{2}\right) & \ldots
\end{array}\right]
$$

Proper Orthogonal Decomposition (POD) is based on the diagonalization of the matrix $X$. The mathematical procedure transforms the number of possibly correlated variables into a smaller number of uncorrelated variables. The first component contains as much of the variation in the system as possible. 


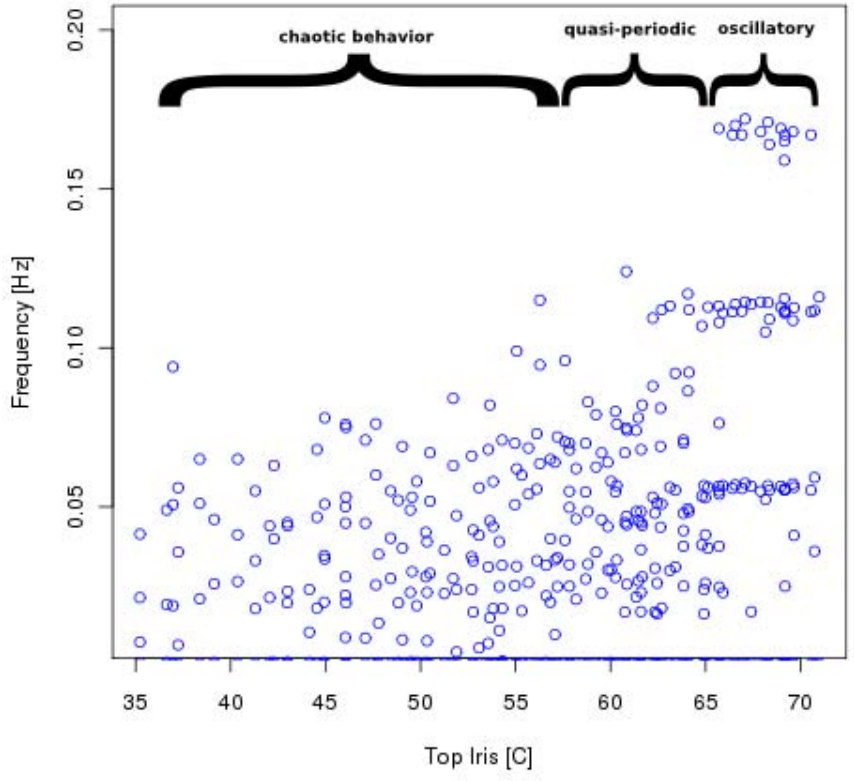

Figure 21 Frequencies measured for $\eta=0.40$ over a range of $\Delta T$ 's (Rayleigh number). Frequency bifurcations occur near $T_{\text {iris }}=$ $66^{\circ} C\left(\Delta T=-6^{\circ} C\right)$.

For the POD analysis the data is centered by the mean of each row. Then the covariance matrix $C_{x}$ is calculated. The covariance matrix is a square, symmetric $m \times m$ matrix whose diagonal represents the variance of particular measurements. Small diagonal terms indicate that the variables are statistically independent.

$$
C_{x}=\frac{1}{n-1} X X^{T}
$$

Singular Value Decomposition (SVD) is used to diagonalize the matrix. The SVD diagonalization is shown in Equation 9, where $U \in$ $\mathbb{C}^{m \times m}$ is unitary, $V \in \mathbb{C}^{n \times n}$ is unitary, and $\Sigma \in \mathbb{R}^{m \times n}$ is diagonal. If full SVD is used for calculations, it may be applied to rank deficient matrices, as shown in Trefethen and Bau (1997).

$$
X=U \Sigma V^{*}
$$

Theorem: Every matrix $X \in \mathbb{C}^{m \times n}$ has a singular value decomposition. Furthermore, the singular values $\left\{\sigma_{j}\right\}$ are uniquely determined, and if $X$ is square and the $\sigma_{j}$ distinct, the singular vectors $\left\{u_{j}\right\}$ and $\left\{v_{j}\right\}$ are uniquely determined up to complex signs (complex scalar factors of absolute value 1).

A visual representation of the SVD process is shown in Fig. 22. The following theorems illustrate the way SVD is linked to POD, where the first theorem confirms that the basis vectors are unique, and the second theorem allows determination of $N$ such that the Nth partial sum captures as much of matrix $X$ as possible.

Theorem: $X$ is the sum of $\mathrm{r}$ rank-one matrices.

$$
X=\Sigma_{j=1}^{r} \sigma_{r} u_{j} v_{j}^{*}
$$

Theorem: For any $N$ such that $0 \leq N \leq r$, the partial sum is defined as:

$$
X=\Sigma_{j=1}^{N} \sigma_{r} u_{j} v_{j}^{*}
$$

And if $N=\min \{m, n\}$, define $\sigma_{N+1}=0$. Then

$$
\left\|X-X_{N}\right\|_{2}=\sigma_{N+1}
$$

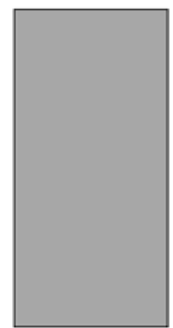

$x$

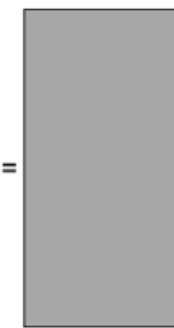

U

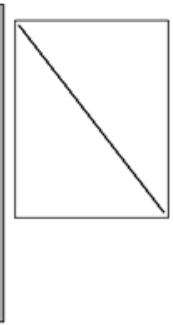

$\Sigma$

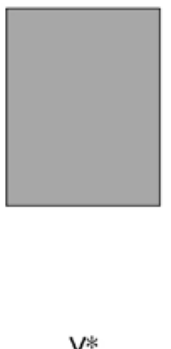

V*
Figure 22 Visual representation of the SVD diagonalization. Adapted from Trefethen and Bau (1997).

SVD provides a type of least-square fitting algorithm, which projects the matrix onto low-dimensional representations in a formal, algorithmic way, Trefethen and Bau (1997). The matrix may also be projected onto other bases as considered by a number of authors listed in Table 2 (projection type is included in the table). For the results presented here, the matrix is projected back onto the modes, to quantify the accuracy of the empirical eigenvalues.

The experimental data was first analyzed as a matrix of only the measured air temperatures, and then subsequently as a matrix combination of air and polymer preform temperatures. Table 4 shows the matrix size for each analysis. For each analysis only one air thermocouple and one preform thermocouple needed to be treated because of the redundancy in the frequency data as determined by evaluating the covariance between the different azimuthally located air and preform thermocouples.

Table 4 Matrix sizes for SVD of the air temperature data alone, and the combined air and preform temperature data.

\begin{tabular}{ccc}
\hline Matrix & Air Data & $\begin{array}{c}\text { Air and } \\
\text { Preform Data }\end{array}$ \\
\hline$X$ & $12 \times 201$ & $24 \times 201$ \\
$U$ & $201 \times 201$ & $201 \times 24$ \\
$\Sigma$ & $201 \times 12$ & $201 \times 24$ \\
$V$ & $12 \times 12$ & $24 \times 24$ \\
\hline
\end{tabular}

\subsection{POD of Air Temperature Data}

Table 5 lists the eigenvalues and energies associated with each of the modes, for the analysis of only the air temperatures. Eight modes are needed to represent 90 percent of the energy. Homes et al. (1996) recommend that the number of modes be determined in order to capture 90 percent of the energy, and furthermore that no excluded mode should contain more than one percent of the remaining energy. In this analysis, only the 90 percent energy threshold was used. Future work could investigate the increased accuracy that would occur by including additional modes.

The magnitudes of the modes are shown in Fig. 23. Fig. 24 compares the behavior of the first mode with the experimental data. (The data has been normalized to make the variations clearer.) Here it is evident that the first mode is insufficient to characterize the actual behavior, as the system transitions from a strongly oscillatory to chaotic regime with increasing $\Delta T$. More than one mode is clearly required to capture the behavior of the system.

The 90 percent threshold is used to determine the total modes required, which in this case is eight. Fig. 25 compares the behavior of the combined first eight modes with the experimental data. Although the excluded modes, 9-12, each contain more than one percent of the energy, 
Table 5 Summary of modes and energy for air temperature analysis.

\begin{tabular}{llll}
\hline mode & $\begin{array}{l}\text { eigenvalue } \\
\sigma\end{array}$ & $\begin{array}{l}\text { Energy } \\
\text { percent }\end{array}$ & $\begin{array}{l}\text { Total Energy } \\
\text { percent }\end{array}$ \\
\hline 1 & 0.0098536 & 18.4415 & 18.4415 \\
2 & 0.0083267 & 15.584 & 34.0255 \\
3 & 0.007322 & 13.7035 & 47.729 \\
4 & 0.0064848 & 12.1367 & 59.8657 \\
5 & 0.0058181 & 10.8889 & 70.7546 \\
6 & 0.0047224 & 8.8382 & 79.5928 \\
7 & 0.0045264 & 8.4714 & 88.0642 \\
8 & 0.0026675 & 4.9924 & 93.0566 \\
9 & 0.0017098 & 3.2 & 96.2566 \\
10 & 0.00080831 & 1.5128 & 97.7694 \\
11 & 0.00062095 & 1.1621 & 98.9315 \\
12 & 0.00057088 & 1.0684 & 100 \\
\hline
\end{tabular}

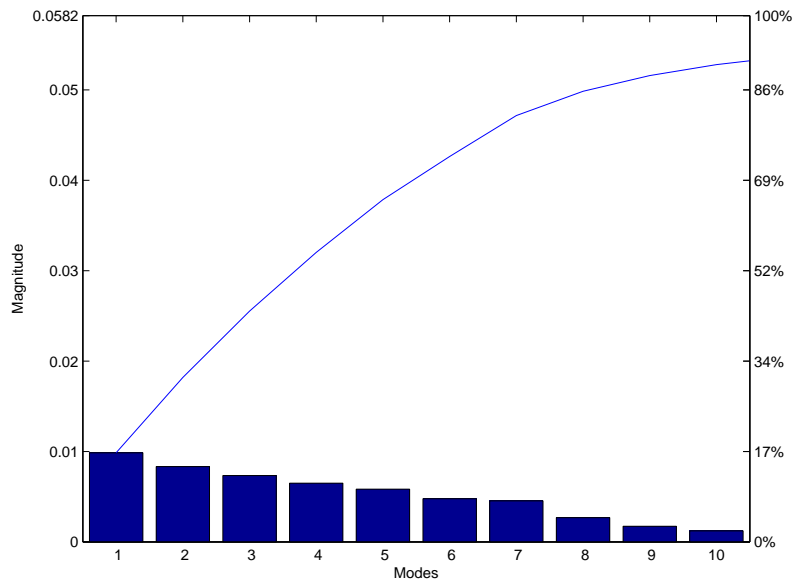

Figure 23 Modes calculated for the air temperature POD analysis.

modes 1-8 capture the important features of the experimental information. For this range of $\Delta T$, there is considerable dynamic information as the system transitions from steady to oscillatory and then to chaotic with increasing $\Delta T$. Even with these transitions, the experimental data is well characterized by the first eight modes.

\subsection{POD of Air and Preform Temperature Data}

Table 6 lists the eigenvalues and energies associated with each of the modes, for the analysis of both the air and preform temperatures. In this case, ten modes are needed to capture 90 percent of the energy. If additional accuracy is desired then the modes containing on the order of one or more percent of the energy could be included, as suggested by Homes et al. (1996), indicating modes up to mode 19 should be included.

The comparison between the behavior based on ten modes and the experimental data is shown in Fig. 26. The use of ten modes (90\% energy) appears to capture most of the air temperature behavior, but somewhat less of the response in the polymer preform temperature. This suggests that more modes are needed to capture the air and preform temperatures simultaneously.

\section{CONCLUSIONS}

Experimental results for natural air convection in an $\eta=0.40, A=20.7$ annular cavity show fully chaotic behavior for $\Delta T>2^{\circ} C$. This is in contrast to previous results in an $\eta=0.60, A=31.0$ cavity which
Table 6 Summary of modes and energy for air and preform temperature analysis.

\begin{tabular}{llll}
\hline mode & eigenvalue & $\begin{array}{l}\text { Energy } \\
\text { percent }\end{array}$ & $\begin{array}{l}\text { Total Energy } \\
\text { percent }\end{array}$ \\
\hline 1 & 0.0098603 & 16.9356 & 16.9356 \\
2 & 0.008334 & 14.3141 & 31.2497 \\
3 & 0.0073384 & 12.6042 & 43.8539 \\
4 & 0.0064926 & 11.1514 & 55.0053 \\
5 & 0.0058349 & 10.0217 & 65.0270 \\
6 & 0.00477 & 8.1928 & 73.2198 \\
7 & 0.0045366 & 7.7918 & 81.0116 \\
8 & 0.0026832 & 4.6086 & 85.6202 \\
9 & 0.0017317 & 2.9743 & 88.5945 \\
10 & 0.001215 & 2.0869 & 90.6814 \\
11 & 0.00086 & 1.4771 & 92.1585 \\
12 & 0.00067928 & 1.1667 & 93.3252 \\
13 & 0.00061279 & 1.0525 & 94.3777 \\
14 & 0.00056793 & 0.97545 & 95.3532 \\
15 & 0.00045438 & 0.78043 & 96.1336 \\
16 & 0.00036096 & 0.61997 & 96.7534 \\
17 & 0.00033485 & 0.57512 & 97.3285 \\
18 & 0.00031727 & 0.54494 & 97.8734 \\
19 & 0.00029244 & 0.50228 & 98.3757 \\
20 & 0.0002242 & 0.38508 & 98.7608 \\
21 & 0.00020404 & 0.35045 & 99.1112 \\
22 & 0.00018467 & 0.31719 & 99.4284 \\
23 & 0.00016704 & 0.28689 & 99.7153 \\
24 & 0.00016562 & 0.28446 & 100 \\
\hline & & & \\
\hline
\end{tabular}

showed no chaotic behavior for the range of $\Delta T$ 's imposed, as high as $\Delta T=45^{\circ} \mathrm{C}$. Furthermore, temperature oscillations in the wider $\eta=$ 0.40 geometry showed much lower amplitudes than those in the narrower $\eta=0.60$ geometry.

In both $\eta=0.40$ and $\eta=0.60$ geometries, as the buoyant driving potential $(\Delta T)$ is increased, the transition from steady to oscillatory flow occurs as a supercritical Hopf bifurcation, and then the system follows a frequency doubling route to chaos. In previous studies of the $\eta=0.60$ geometry, frequency bifurcation was very clear; however, the imposed driving potential $(\Delta T)$ was not sufficiently large to produce fully chaotic flow. By contrast, fully chaotic flow was readily achieved with imposed $\Delta T$ as low as $2^{\circ} C$ in the $\eta=0.40$ system, but the transition from oscillatory to chaotic behavior occurred over such a narrow change in $\Delta T$ that frequency bifurcation was much less clear in this wider annular geometry.

For the $\eta=0.40$ geometry, the two strongest air temperature frequencies are at 0.06 and $0.11 \mathrm{~Hz}$ in the oscillatory regime, and at 0.05 $\mathrm{Hz}$ in the quasi-periodic regime. These same frequencies are respectively echoed in the polymer preform temperatures, for the oscillatory and quasi-periodic flow regimes.

For the oscillatory regime of $\eta=0.40$, air temperature variations were measured over a range of vertical $\mathrm{z}$ locations. These measurements suggest the probable existence of distinct and multiple flow cells in the oscillatory regime.

In the natural convection systems studied here, a wide dynamic range is explored over two flow transitions from steady to oscillatory and then to chaotic. For such systems, the POD modes do not show strong exponential decay, as in previous studies from the literature. In previous results with a smaller number of modes the analyses were not based on transition systems. Other results from the literature required far fewer modes to accurately capture the system behavior; however, in such cases, Sahan (2000), the authors analyzed only one flow transition rather than two. 

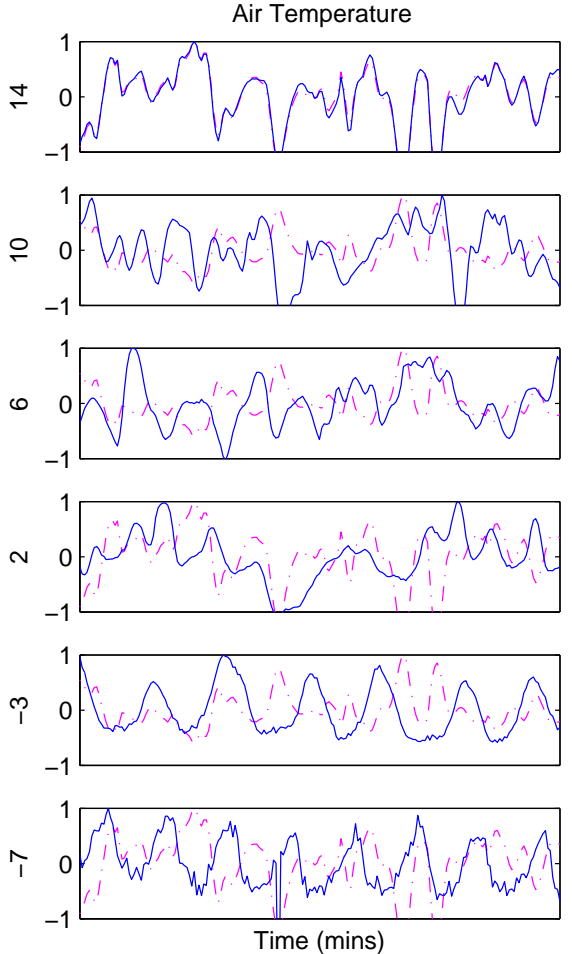
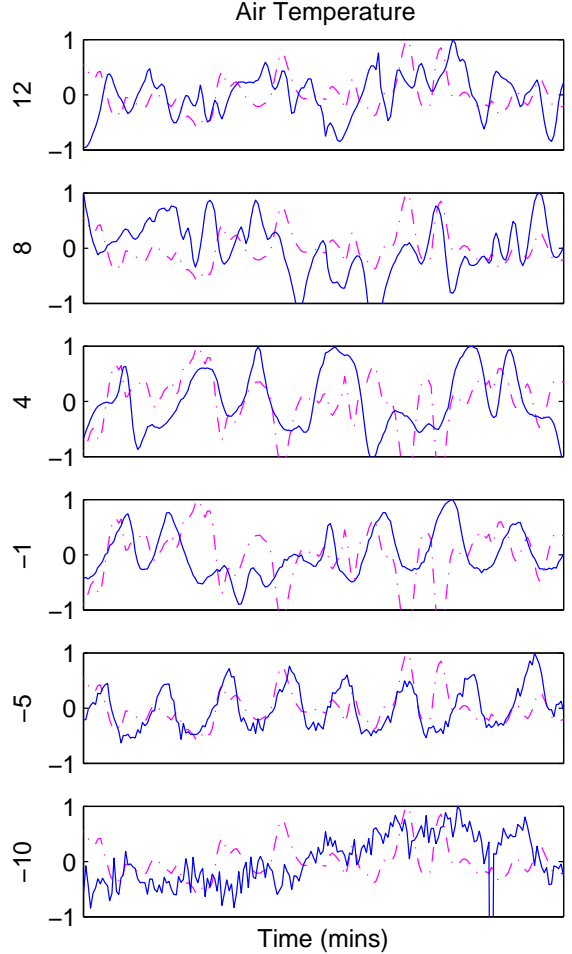

Figure 24 First mode (dashed) projected onto the experimental air temperature data (solid) for $\eta=0.40$. The data has been normalized to a unit scale to make the variations more clear. The experimentally imposed $\Delta T$ is shown at the left margin for each data set.
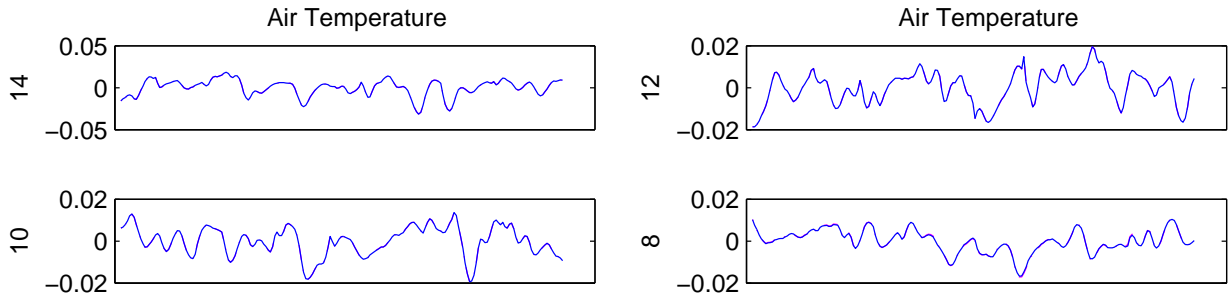

${ }_{-0.02}^{0.02}$
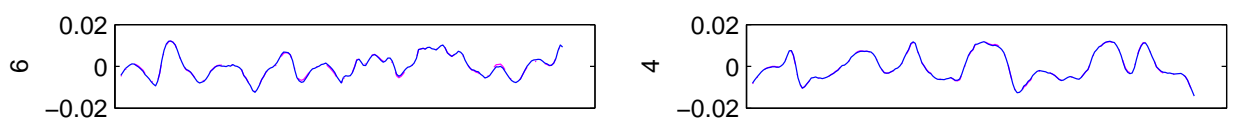

N
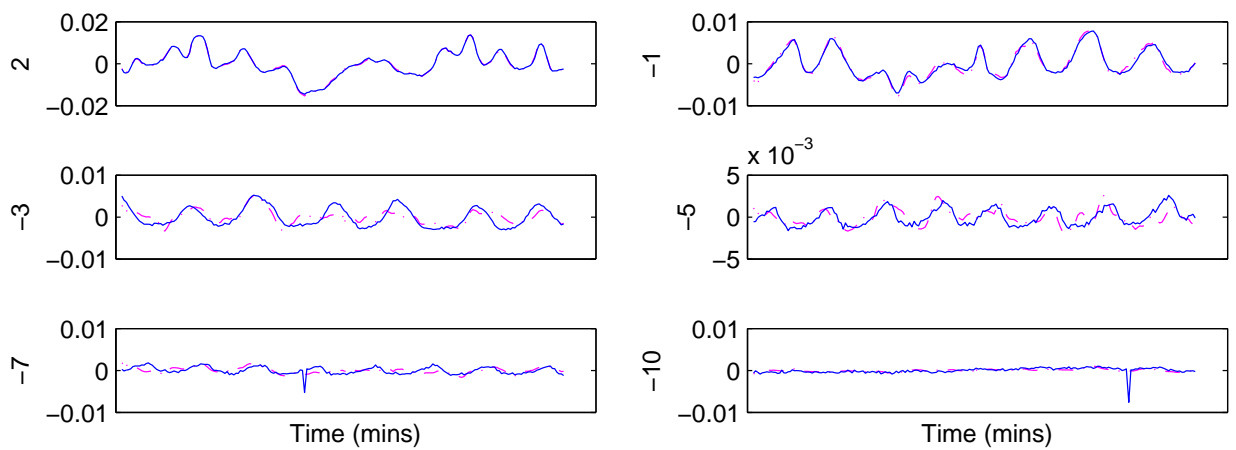

Figure 25 Combined first eight modes (dashed) projected onto the experimental air temperature data (solid) for $\eta=0.40$. The data has not been normalized to emphasize that the magnitudes of the errors are small. The experimentally imposed $\Delta T$ is shown at the left margin for each data set. 
Table 7 summarizes the number of modes required in the POD analyses to capture 90 percent of the energy in the $\eta=0.40, A=20.7$ annular air system. In the first case, only air temperatures are used in the POD analysis and eight modes are required to meet the 90 percent energy threshold. Comparisons with the original experimental data show that the projected modes adequately capture air temperature behavior in the chaotic regime, but are less successful in the oscillatory and steady regimes. In the second case, both air and preform temperatures are used in the POD analysis, and ten modes are required to meet the 90 percent energy threshold.

Table 7 Summary of POD results for air temperature data only and combined air and preform temperature data.

\begin{tabular}{|l|c|c|}
\hline$\Delta T\left[{ }^{\circ} C\right]$ & $\begin{array}{c}\text { Experimental } \\
\text { Air Data }\end{array}$ & $\begin{array}{c}\text { Experimental Air } \\
\text { and Preform Data }\end{array}$ \\
$\begin{array}{l}\text { Flow } \\
\text { Regimes }\end{array}$ & $\begin{array}{c}\text { chaotic, } \\
\text { oscillatory } \\
\text { and steady }\end{array}$ & $\begin{array}{c}\text { chaotic, } \\
\text { oscillatory } \\
\text { and steady }\end{array}$ \\
Modes & 8 & 10 \\
\hline
\end{tabular}

\section{ACKNOWLEDGEMENTS}

The authors express sincere appreciation to the National Science Foundation for the principal funding of this work through Grant 0626533. Special thanks to Elliott Schmitt and Ethan Lorimor for technical review. Many undergraduate students assisted in the development of the experimental work presented here including Nick Stelzenmuller, Jaeger Dill, Kimberley Hartman, and Owen Robertson.

\section{NOMENCLATURE}

A Aspect ratio

$c_{p} \quad$ specific heat

$H \quad$ annulus height

$k \quad$ thermal conductivity

PIV Particle Image Velocimetry

POD Proper Orthogonal Decomposition

POF Polymer Optical Fiber

Pr Prandlt Number

$R \quad$ radius

$\mathrm{Ra} \quad$ Rayleigh Number

sdf Spectral Density Function

SVD Singular Value Decomposition

$t \quad$ time

$T \quad$ temperature

$r \quad$ radial coordinate

WOSA Welch's Overlapped Segment Averaging

$z \quad$ vertical coordinate

Greek Symbols

$\rho$ density

$\eta \quad$ radius ratio

$\Delta T \quad$ temperature difference $\left(T_{\text {peak }}-T_{\text {iris }}\right)$

\section{Subscripts}

c critical

$i \quad$ inner

o outer

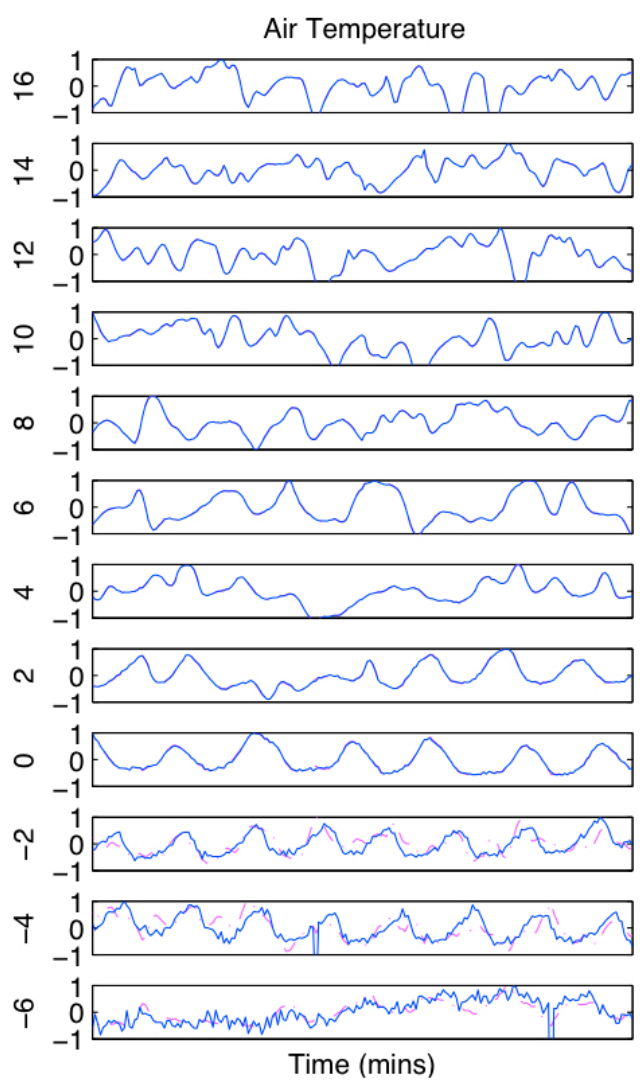

Preform Temperature

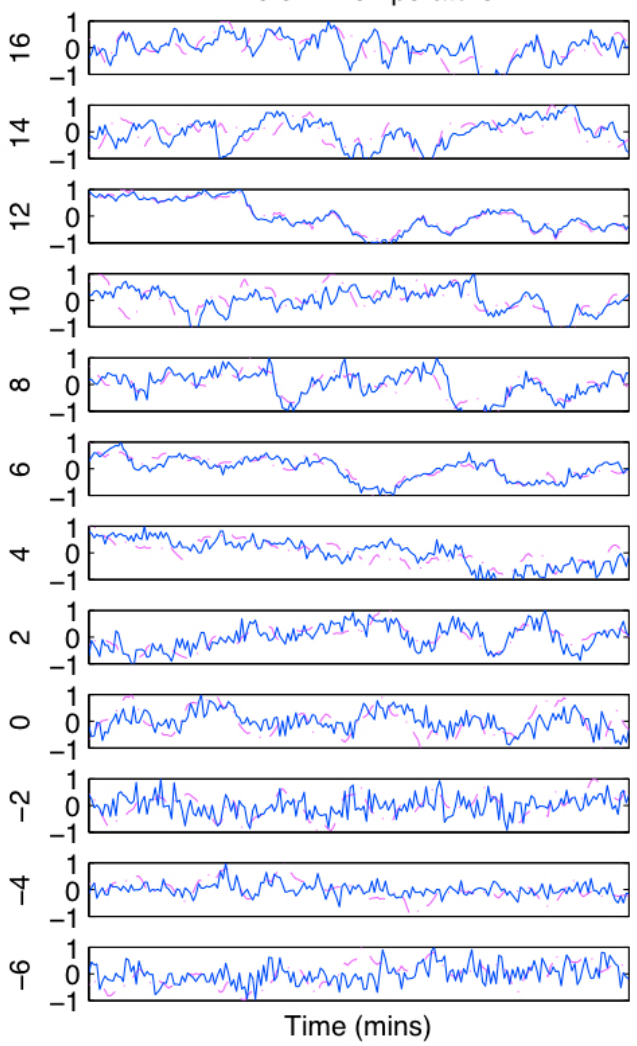

Figure 26 Combined first ten modes (dashed) projected onto experimental air and preform temperature data (solid) for $\eta=0.40$. The data has been normalized to a unit scale to make the variations more clear. The experimentally imposed $\Delta T$ is shown at the left margin for each data set. 


\section{REFERENCES}

Asokan, B., and Zabaras, N., 2005, "Using Stochastic Analysis to capture unstable equilibrium in natural convection," Journal of Compuational Physics, 208, 134-153, doi:10.1016/j.jcp.2005.02.007.

Ball, K., Sirovich, L., and Keefe, L., 2005, "Dynamical eigenfunction decomposition of turbulent channel flow," International Journal for $\mathrm{Nu}$ merical Methods in Fluids, 12(6), 585-604.

Blinov, D., Prokopov, V., Sherenkovskii, Y., Fialko, N., and Yurchuk, V., 2004, "Effective Method for Construction of Low-dimensional Models for Heat Transfer Process," International Journal Heat and Mass Transfer, 47, 5823-5828,

doi:10.1016/j.ijheatmasstransfer.2004.07.020.

Bonnet, J., Cole, D., Delville, J., Glauser, M., and Ukeiley, L., 1994, "Stochastic estimation and proper orthogonal decomposition: Complementary techniques for identifying structure," Experiments in Fluids, 17, $307-314$,

doi:10.1007/BF01874409.

Choi, I., and Korpela, S., 1980, "Stability of the conduction regime of natural convection in a tall vertical annulus," Journal of Fluid Mechanics, 99, 725-738,

doi:10.1017/S0022112080000869.

Citriniti, J., and George, W., 2000, "Reconstruction of the Global Velocity Field in the Axisymmetric Mixing Layer Utilizing the Proper Orthogonal Decomposition," Journal of Fluid Mechanics, 418, 137-166, doi:10.1017/S0022112000001087.

Dillon, H., Emery, A., and Mescher, A., 2009, "Flow instabilities from a Parabolic Wall Profile in an Annular Cavity," ASME IMECE, (IMECE2009-11657), doi:10.1115/IMECE2009-11657.

Dillon, H., 2011, Chaotic Natural Convection in an Annular Geometry, Ph.D. thesis, University of Washington.

Homes, P., Lumley, J.L., and Berkooz, G., 1996, Turbulence, Coherent Structures, Dynamical Systems and Symmetry, Cambridge University Press.

Keyhani, M., Kulacki, F., and Christensen, R., 1983, "Free convection in a vertical annulus with constant heat flux on the inner wall," Journal of Heat Transfer, 105, 454-459,

doi:10.1115/1.3245606.

Liakopoulos, A., Blythe, P., and Gunes, H., 1997, "A Reduced Dynamical Model of Convective Flows in Tall Laterally Heated Cavities," Proc R Soc London A, 453, 663-672,

doi:10.1098/rspa.1997.0037.

Liang, Y., Lee, H., Lim, S., Lin, W., Lee, K., and Wu, C., 2002, "Proper Orthogonal Decomposition and its Applications - Part I: Theory," Journal of Sound and Vibration, 252(3), 527-544.
Ly, H., and Tran, H., 2001, "Modeling and Control of Physical Processes using Proper Orthogonal Decomposition," Journal of Mathematical and Computer Modeling, 33(1-3), 223, doi:10.1016/S0895-7177(00)00240-5.

Park, H., and Jung, W., 2001, "The Karhunen-Loeve Galerkin Method for the Inverse Natural Convection problems," International Journal Heat and Mass Transfer, 44, 155-167,

doi:10.1016/S0017-9310(00)00092-2.

Podvin, B., and Quere, P.L., 2001, "Low-Order Models for the Flow in a Differentially Heated Cavity," Physics of Fluids, 13(11), 3204, doi:10.1063/1.1408919.

Reeve, H., 2003, Effect of Natural Convection Heat Transfer During Polymer Optical Fiber Drawing, Ph.D. thesis, University of Washington.

Sahan, R., 2000, "Low-Order Dynamical Modeling of Natural Convective Air-Cooling System in a Vertical Channel," IEEE, 140, doi:10.1109/ITHERM.2000.866183. Inter Society Conference on Thermal Phenomena.

Sirovich, L., Ball, K., and Handler, R., 1991, "Propagating Structures in Wall-Bounded Turbulent Flows," Theoretical and Computational Fluid Dynamics, 2, 307-317, doi:10.1007/BF00271470.

Stogatz, S., 2000, Nonlinear Dynamics and Chaos, Perseus Books Publishing.

Stork, K., and Muller, U., 1975, "Convection in boxes: an experimental investigation in vertical cylinders and annuli," Journal of Fluid Mechanics, 71, 231-240,

doi:10.1017/S0022112075002534.

Tarman, H., 2003, "A Karhunen-Loeve Based Approach to Numerical Simulation of Transition in Rayleigh-Benard Convection," Numerical Heat Transfer, Part B, 43, 567-586, doi: $10.1080 / 713836315$.

Tarman, I., 1996, “A Karhunen-Loeve analysis of Turbulent Thermal Convection," International Journal for Numerical Methods in Fluids, 22, 67-79,

doi:10.1002/(SICI)1097-0363(19960115)22:1<67::AID-

FLD332>3.0.CO;2-C.

Trefethen, L.N., and Bau, D., 1997, Numerical Linear Algebra, Society for Industrial and Applied Mathematics.

Webber, G., Handler, R., and Sirovich, L., 1997, "The Karhunen-Loeve decomposition of minimal channel flow," Phys Fluids, 9(4), 1054, doi:10.1063/1.869362.

Weidman, P.D., and Mehrdadtehranfar, G., 1983, "Instability of natural convection in a tall vertical annulus," Physics of Fluids, 105, 454-459.

Yiqin, Y., Huiling, C., Hua, Y., and Lin, Q., 1995, "Determintation of optimum annular gap for minimum heat losses," 1995 National Heat Transfer Conference ASME, 305(3), 99. 